Review

\title{
A Systematic Review and Meta-Analysis of Wound Complications after a Caesarean Section in Obese Women
}

\author{
Aneta Słabuszewska-Jóźwiak ${ }^{1, * \mathbb{C}}$, Jacek Krzysztof Szymański ${ }^{1}{ }^{(D)}$, Łukasz Jóźwiak ${ }^{2}$ and Beata Sarecka-Hujar ${ }^{3}$ (D) \\ 1 First Department of Obstetrics and Gynaecology, Centre of Postgraduate Medical Education, ul. Żelazna $90 \mathrm{~s}$, \\ 01-004 Warsaw, Poland; jkszymanski2@gmail.com \\ 2 Department of Obstetrics and Gynaecology, St. Sofia Hospital, ul. Żelazna 90, 01-004 Warsaw, Poland; \\ luk2jozwiak@gmail.com \\ 3 Department of Basic Biomedical Science, Faculty of Pharmaceutical Sciences in Sosnowiec, \\ Medical University of Silesia, ul. Kasztanowa 3, 41-200 Sosnowiec, Poland; beatasarecka@poczta.onet.pl \\ * Correspondence: anetaslabuszewska@gmail.com; Tel.: +48-504-187-297
}

Citation: Słabuszewska-Jóźwiak, A.; Szymański, J.K.; Jóźwiak, Ł.;

Sarecka-Hujar, B. A Systematic

Review and Meta-Analysis of Wound Complications after a Caesarean Section in Obese Women. J. Clin. Med. 2021, 10, 675. https://doi.org/ $10.3390 /$ jcm10040675

Academic Editors: Grzegorz Jakiel and Ferdinando Antonio Gulino

Received: 27 December 2020

Accepted: 7 February 2021

Published: 10 February 2021

Publisher's Note: MDPI stays neutral with regard to jurisdictional claims in published maps and institutional affiliations.

Copyright: (C) 2021 by the authors. Licensee MDPI, Basel, Switzerland. This article is an open access article distributed under the terms and conditions of the Creative Commons Attribution (CC BY) license (https:/ / creativecommons.org/licenses/by/ $4.0 /)$.

\begin{abstract}
Background: Caesarean sections in obese patients are associated with an increased risk of surgical wound complications, including hematomas, seromas, abscesses, dehiscence, and surgical site infections. The aim of the present study is to perform a meta-analysis and systematic review of the current literature focusing on the strategies available to decrease wound complications in this population. (2) Methods: We reviewed the data available from the PubMed and the Science Direct databases concerning wound complications after caesarean sections in obese women. The following key words were used: "caesarean section", "cesarean section", "wound complication", "wound morbidity", and "wound infection". A total of 540 papers were retrieved, 40 of which were selected for the final systematic review and whereas 21 articles provided data for meta-analysis. (3) Results: The conducted meta-analyses revealed that the use of prophylactic drainage does not increase the risk of wound complications in obese women after a caesarean sections (pooled OR $=1.32 ; 95 \% \mathrm{CI}$ $0.64-2.70, p=0.45$ ) and that vertical skin incisions increase wound complications (pooled $\mathrm{OR}=2.48$; 95\% CI 1.85-3.32, $p<0.01$ ) in obese women, including extremely obese women. (4) Conclusions: Subcutaneous drainage does not reduce the risk of a wound complications, wound infections, and fever in obese women after caesarean sections. Negative prophylactic pressure wound therapy (NPWT) may reduce the risk of surgical site infections. The evidence of using a prophylactic dose of an antibiotic before the caesarean section is still lacking.
\end{abstract}

Keywords: caesarean section; wound complication; wound morbidity; wound infection; surgical-site infection

\section{Introduction}

Obesity is a chronic disease that leads to the development of metabolic disorders and cardiovascular complications and currently poses a challenge for healthcare systems around the world [1]. According to the World Health Organisation (WHO), the problem of obesity is reaching epidemic proportions, in both developed as well as developing countries. In 2016, 15\% of women over 18 years of age were either overweight or obese [2]. This problem also affects $1 / 3$ of women of reproductive age [3], including $13 \%$ of pregnant women [4]. The rising prevalence of weight gain during pregnancy is associated with the occurrence of a greater number of complications during pregnancy, childbirth, or the postpartum period [5]. Research confirms that being overweight may also increase the rate of complications in both pregnant women and newborn babies [6]. Obese women develop arterial hypertension [7] and diabetes more often in pregnancy and undergo caesarean sections significantly more often [8], which means that they are diagnosed with postoperative surgical wound healing disorders more frequently [9].

Postoperative complications include: superficial infections, dehiscence, or the presence of a fluid reservoir (seroma and hematoma) at the wound site. The above symptoms 
concern 3 to $15 \%$ of women after a caesarean section $[10,11]$ and often result in prolonged hospitalization, antibiotic therapy, thus leading to increased postpartum care costs. A superficial infection is part of a surgical site infection (SSI) which, according to the Centers for Disease Control and Prevention (CDC), is an infection that occurs within 30 days of the performed surgical procedure. The risk factors for the above complications include young age at childbirth, smoking, obesity, arterial hypertension, diabetes, chorioamnionitis, increased intrapartum blood loss, prolonged ruptured of membrane, emergency caesarean section and subsequent surgical delivery, use of suboptimal antibiotic prophylaxis, improper preparation of the surgical field, extended duration of the surgical procedure, and the employed caesarean section technique, including that of the incision and of the suturing of the skin [12-15].

The aim of the study is to review the current literature in order to determine the factors influencing the healing of postoperative wounds in obese women undergoing a caesarean section.

\section{Materials and Methods}

This systematic review was conducted in accordance with the Preferred Reporting Items for Systematic Reviews and Meta-Analyses (PRISMA) guideline [16].

\subsection{Search Strategy}

We searched the following databases: PubMed, Science Direct Web of Science as well as Google Scholar for relevant papers published from 2010 to 2020 (last search in October 2020). The small number of studies on subcutaneous drainage in this period contributed to an extended search strategy in this field for the years 2000-2020. The following key words were used: "caesarean section," "cesarean section," "wound complication," "wound morbidity," "wound infection." Two authors (A.S.-J., B.S.-H.) independently reviewed the search results to identify relevant studies and disagreements between the reviewers were arbitrated. The study titles and abstracts were screened according to the following inclusion/exclusion criteria. The references cited in the found articles were also searched in order to identify other published articles on the topic. The selected relevant studies selected were classified according to the population, intervention, comparison, and outcome (PICO) framework in order to identify the relevant research questions meeting the following selection criteria:

Population: obese women;

Intervention: caesarean section;

Comparison: women with normal weight vs. overweight women;

Outcomes: wound infection, wound complications, and wound morbidity.

Separate searches were performed for each of the topics covered in this review followed by the deletion of any duplicates. At the first step of elimination, the studies that were clearly irrelevant based on their title were removed. Next, the remaining abstracts were reviewed and those that were irrelevant to the topic were excluded. The remaining papers were comprehensively read to determine whether they contain the relevant information.

\subsection{Inclusion and Exclusion Criteria}

Retrospective, prospective control, and randomized cohort studies reporting wound complication after elective, emergency or intrapartum caesarean sections in obese women, who were defined as women with a body mass index (BMI) of $\geq 30 \mathrm{~kg} / \mathrm{m}^{2}$ before pregnancy or at the time of delivery, were considered. Studies available in English with a publication date ranging from 2010 to 2020 and available in English describing the placement of a skin incision, wound dressing, or skin closure was included into the final analysis.

The exclusion criteria were a caesarean section performed before 23 weeks of pregnancy and participants aged under 18 or over 45 years. Reviews, commentaries, and case reports were also excluded. 


\subsection{Selection Process and Result Codification}

The search, selection, and analysis of the studies found during the literature search were conducted independently by two team members. The selection was based on reading the title and abstract, followed by the full text of the paper and, finally, a reverse search in the selected studies. Outcomes were considered clearly defined if the authors provided an adequate level of detail about of criteria. Disagreements were resolved through arbitration and discussion with other authors. For the meta-analysis, we selected studies that used the same measuring instrument and provided the data necessary for its execution.

\subsection{Critical Reading and Level of Evidence}

The studies included in the research were reviewed critically for bias analysis using the Strengthening the Reporting of Observational Studies in Epidemiology (STROBE) checklist [17]. The selected studies were assigned a methodological quality grade according to the levels of evidence and degrees of recommendation proposed by the Jadad scale [18] (Supplementary Table S1).

\subsection{Statistical Analysis}

Statistical analyses were performed using the Review Manager software (RevMan version 5.4 Cochrane, London, UK) and MedCalc software (version 19.5.3.; MedCalc Software Ltd., Ostend, Belgium). The pooled odds ratio (OR) along with a $95 \%$ confidence interval (CI) for the following comparisons: women with a drain vs. women without a drain, women with vertical skin incision vs. women with transverse skin incision, women with NPWT vs. women with standard therapy in terms of wound complications, infections, as well as fever were calculated. The results of the heterogeneity between studies, i.e., the $\mathrm{I}^{2}$ test at level of $50 \%$, allowed to use between the random effects model (REM) or the fixed effects model (FEM). $\mathrm{I}^{2}$ expresses the proportion of dispersion due to heterogeneity and $\mathrm{I}^{2}$ at $25 \%, 50 \%$, and $75 \%$ was suggested as low, intermediate, and high inconsistency. The publication bias was evaluated both visually with inspection of funnel plots and by performing Egger's test as well as Begg's test; however according to the recommendations, at least 10 studies must be included to conduct funnel plot asymmetry tests so as to maintain sufficient power for distinguishing the chance from real asymmetry $[19,20]$. In addition, the sensitivity analyses were performed by omitting each included study at a time (the leave-one-out method) to reflect the influence of the individual data set to the pooled OR and in consequence to assess whether the results remained stable and reliable. The results of the carried out meta-analyses were summarized in tables and illustrated using forest plots.

\section{Results}

\subsection{Study Selection Process}

A total of 540 articles were first selected. For the initial screening, 30 duplicates were identified and removed, leaving 510 articles. The titles and abstracts were then assessed by two reviewers, ending with the inclusion of 94 articles. The full texts were then retrieved for those citations that were considered potentially relevant and assessed in terms of their eligibility by the two reviewers. Of these 94 articles, 34 were excluded. The most common reasons for the exclusions were case reports and a language other than English. The reference lists of the included studies were hand searched by the first author. Separate searches were performed for each of the topics covered in this review. The first elimination step involved removing, the studies that were clearly irrelevant based on their title followed by excluding any duplicates. Next, the remaining abstracts were reviewed and the irrelevant ones in terms of the topic were removed. The full text of the paper was read for all the remaining papers in order to determine whether they contained the relevant information.

Eventually, a total of 40 relevant articles $[10,11,21-58]$ were included in the current systematic literature review whereas 21 articles provided data for meta-analysis. Six studies re- 
garding skin incision $[24,26,27,31,33,42]$ and 9 studies regarding drainage $[21-25,27,32,35,46]$ were performed in meta-analysis. The frequency of surgical site infection (SSI) in obese women with negative pressure wound therapy (NPWT) in comparison to women with standard therapy was based on 6 studies $[36,37,39,43,45,47]$. A summary of the search process is illustrated in Figure 1, as recommended by the PRISMA guidelines [16].
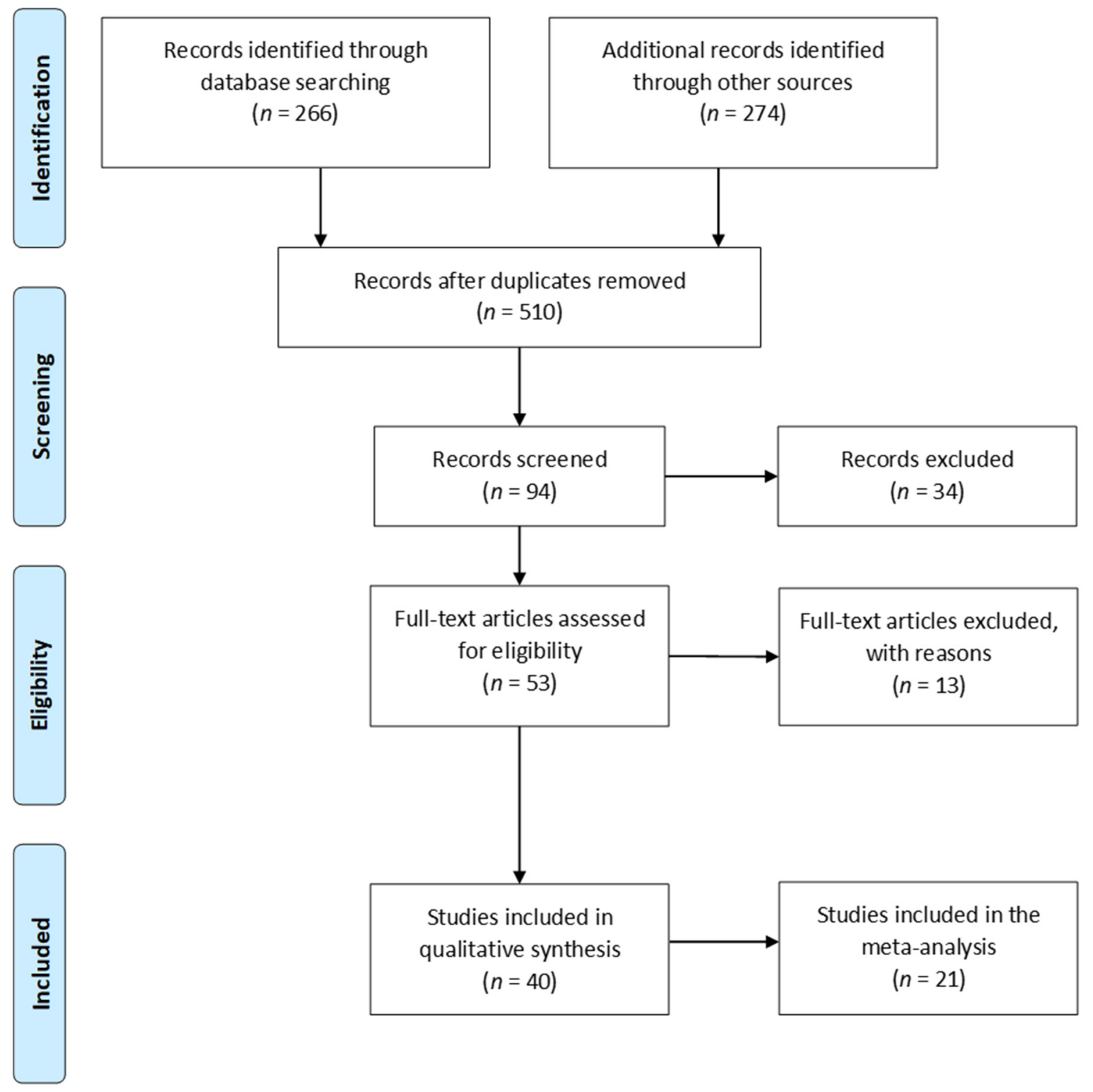

Figure 1. Flow chart presenting the process of searching for eligible articles according to PRISMA guidelines.

\subsection{Characteristics of Included Studies}

The studies included in the systematic review are retrospective cohort studies or a secondary analysis of observational cohort studies [21-58] and concern factors that have a potential impact on wound healing in obese patients (BMI $\geq$ he $\mathrm{kg} / \mathrm{m}^{2}$ ) subjected to elective, unplanned, or intrapartum caesarean section. The factors influencing the proper healing processes include concomitant diseases such as arterial hypertension, diabetes, and smoking, as well as the premature rupture of membranes, an infection of the amniotic sac, the method of incision (vertical or transverse), and skin suturing (staplers, intradermal sutures, running, or intermittent sutures), and the use of prophylactic vac dressings. When assessing the effects of the surgical procedure, the authors reported complications including: 
wound infections, endometritis, the presence of a hematoma or serous exudate, and the discontinuation (dehiscence) of the wound edges that are the cause of rehospitalization, which was analyzed in six studies [24,26,27,31,33,42]. In evaluating the effectiveness of vac dressings, the primary goal was to assess the presence of a superficial incisional surgical site infection (SSI). Eleven studies assessed the use of cefazolin in perioperative prophylaxis [48-58], six of which were prospective [48,52,54-58], and two were randomized and double-blind $[51,53]$.

The research was carried out in ten different countries, on four continents, mainly in North America. Twenty-three studies were conducted in the United States [10,11,28, 29,31-34,36,39,40,42,43,46-48,50-53,55-57], and the remaining studies were carried out in Egypt [22,30,32,41], Denmark [44], India [35], Japan [25], Canada [54], Australia [21,58], and New Zealand [49]. Moreover, one study concerned women living in England or Ireland [38] and one in Scotland [46]. Most of the studies included both elective and unplanned caesarean sections $(n=14)$ with the exception of three studies that included emergency caesarean sections as the exclusion criterion [29-31]. In five cases, the surgical procedure was generally referred to as a caesarean section $[10,26,34,38,41]$.

The degree of obesity was defined in accordance with the WHO definition across all studies, distinguishing obesity of the first (BMI $30.0-34.9 \mathrm{~kg} / \mathrm{m}^{2}$ ), the second (BMI $\left.35.0-39.9 \mathrm{~kg} / \mathrm{m}^{2}\right)$, and the third stage $\left(\mathrm{BMI} \geq 40 \mathrm{~kg} / \mathrm{m}^{2}\right)$ [2]. Eight of the analyzed studies only concerned complications in women with morbid obesity, that is, with a BMI of distinguish $^{2}[24,31,33,36,40,42,43,45]$.

Tables 1 and 2 describe the full characteristics of the studies, including the place, the type of study, the size of the group, and the primary goals and results.

Table 1. Characteristics of studies included in the systematic review and meta-analysis.

\begin{tabular}{|c|c|c|c|c|c|c|c|}
\hline Study & Date & Country & Study Design & $N$ & Exclusion Criteria & Definition of Obesity & Primary Outcomes \\
\hline $\begin{array}{l}\text { Magann et al., } \\
2002 *[21]\end{array}$ & 1998-2001 & Australia & $\begin{array}{l}\text { Prospective } \\
\text { randomized study }\end{array}$ & 964 & $\begin{array}{l}\text { - Declined participation in } \\
\text { investigation, } \\
\text { - Emergency caesarean } \\
\text { section without consent to } \\
\text { participate in the study }\end{array}$ & $\begin{array}{l}\text { At least } 2 \mathrm{~cm} \text { of } \\
\text { subcutaneous fat tissue } \\
\text { on admission } \\
\text { to delivery }\end{array}$ & $\begin{array}{l}\text { Risk of wound } \\
\text { disruption after } \\
\text { caesarean delivery }\end{array}$ \\
\hline $\begin{array}{l}\text { Al-Inany et al., } \\
2002 *[22]\end{array}$ & 1999-2000 & Egypt & $\begin{array}{l}\text { Prospective controlled } \\
\text { clinical study }\end{array}$ & 118 & $\begin{array}{l}\text { - Prolonged premature } \\
\text { rupture of membranes, } \\
\text { - Prolonged labor, } \\
\text { - Long preoperative } \\
\text { hospitalization, } \\
\text { - Malignancy, } \\
\text { - Diabetes mellitus, } \\
\text { - Chronic lung disease }\end{array}$ & $\begin{array}{c}\mathrm{BMI}>32 \mathrm{~kg} / \mathrm{m}^{2} \\
\text { subcutaneous fat of at } \\
\text { least } 2 \mathrm{~cm}\end{array}$ & $\begin{array}{l}\text { The incidence of wound } \\
\text { breakdown in both } \\
\text { groups together with } \\
\text { the rate of hematoma } \\
\text { formation and } \\
\text { occurrence of fever }\end{array}$ \\
\hline $\begin{array}{l}\text { Ramsey et al., } \\
2005 *[23]\end{array}$ & 2001-2004 & USA & Randomized study & 280 & $\begin{array}{l}\text { - Inability to obtain informed } \\
\text { consent, } \\
\text { - Moribund caesarean } \\
\text { delivery required, } \\
\text { - No plan for follow-up }\end{array}$ & $\begin{array}{c}\mathrm{BMI}>30 \mathrm{~kg} / \mathrm{m}^{2} \\
\text { on admission }\end{array}$ & $\begin{array}{l}\text { Composite wound } \\
\text { morbidity rate, defined } \\
\text { as any of the following } \\
\text { noted during the } \\
\text { post-hospital discharge } \\
\text { wound follow-up } \\
\text { assessments: } \\
\text { subcutaneous } \\
\text { dehiscence }(1 \mathrm{~cm}), \\
\text { seroma, hematoma, } \\
\text { abscess, or } \\
\text { fascial dehiscence }\end{array}$ \\
\hline $\begin{array}{l}\text { Alanis et al., } \\
2010 *[24]\end{array}$ & 2005-2009 & USA & $\begin{array}{l}\text { Retrospective } \\
\text { cohort study }\end{array}$ & 194 & - Maternal death & $\begin{array}{c}\text { Pre-delivery } \\
\mathrm{BMI} \geq 50 \mathrm{~kg} / \mathrm{m}^{2}\end{array}$ & $\begin{array}{l}\text { Wound complications } \\
\text { (wound disruption, } \\
\text { cellulitis, NOT } \\
\text { superficial wound }\end{array}$ \\
\hline
\end{tabular}


Table 1. Cont.

\begin{tabular}{|c|c|c|c|c|c|c|c|}
\hline Study & Date & Country & Study Design & $N$ & Exclusion Criteria & Definition of Obesity & Primary Outcomes \\
\hline $\begin{array}{l}\text { Inotsume-Kojima } \\
\text { et al., } 2011^{*} \text { [25] }\end{array}$ & 2006-2009 & Japan & $\begin{array}{l}\text { Retrospective } \\
\text { cohort study }\end{array}$ & 71 & $\begin{array}{c}\text { - Informed consent for the } \\
\text { new surgical method was } \\
\text { not } \\
\text { obtained preoperatively, } \\
\text { - A Pfannenstiel incision } \\
\text { was made, } \\
\text { - Surgery revealed } \\
\text { malignancy, } \\
\text { - Subcutaneous fat } \\
\text { thickness was }<2 \mathrm{~cm}, \\
\text { - Hospitalization before } \\
\text { surgery was longer } \\
\text { than } 24 \text { h, } \\
\text { - Surgery was performed } \\
\text { in response to } \\
\text { premature labor, } \\
\text { - Patient was not a native } \\
\text { of Japan }\end{array}$ & $\begin{array}{l}\mathrm{BMI} \geq 28 \mathrm{~kg} / \mathrm{m}^{2} \text { at the } \\
\text { time of admission }\end{array}$ & Wound complications \\
\hline $\begin{array}{l}\text { McLean et al., } \\
2011 \text { [26] }\end{array}$ & 1998-2005 & USA & $\begin{array}{l}\text { Retrospective } \\
\text { cohort study }\end{array}$ & 242 & $\begin{array}{l}\text { Incomplete } \\
\text { medical reports }\end{array}$ & $\begin{array}{c}\mathrm{BMI} \geq 30 \mathrm{~kg} / \mathrm{m}^{2} \\
\text { at delivery }\end{array}$ & $\begin{array}{l}\text { Type of skin incision } \\
\text { and partial or complete } \\
\text { wound separation }\end{array}$ \\
\hline $\begin{array}{l}\text { Thornburg et al., } \\
2012 *[27]\end{array}$ & 1994-2008 & USA & $\begin{array}{l}\text { Retrospective } \\
\text { cohort study }\end{array}$ & 623 & $\begin{array}{l}\text { - Prior caesarean delivery, } \\
\text { - Skin incision other than } \\
\text { vertical or low transverse, } \\
\text { - Missing prepregnancy } \\
\text { height or weight, or } \\
\text { unavailable } \\
\text { follow-up data }\end{array}$ & $\begin{array}{c}\mathrm{BMI} \geq 35 \mathrm{~kg} / \mathrm{m}^{2} \\
\text { at delivery }\end{array}$ & $\begin{array}{l}\text { Presence of any wound } \\
\text { complication defined as } \\
\text { a wound separation, } \\
\text { including both } \\
\text { spontaneous and } \\
\text { indicated as resulting } \\
\text { from seroma formation } \\
\text { or wound } \\
\text { infection/cellulitis }\end{array}$ \\
\hline $\begin{array}{l}\text { Subramanian } \\
\text { et al., } 2014 \text { [28] }\end{array}$ & 2009-2010 & USA & $\begin{array}{l}\text { Retrospective } \\
\text { cohort study }\end{array}$ & 340 & $\begin{array}{l}\text { - Primary wound } \\
\text { infections (purulent } \\
\text { drainage, cellulitis, } \\
\text { and/or abscess requiring } \\
\text { antibiotics or } \\
\text { surgical treatment) }\end{array}$ & $\begin{array}{l}\mathrm{BMI} \geq 30 \mathrm{~kg} / \mathrm{m}^{2} \\
\text { at delivery }\end{array}$ & $\begin{array}{c}\text { Risk factors for } \\
\text { non-infectious wound } \\
\text { disruption following a } \\
\text { caesarean delivery }\end{array}$ \\
\hline $\begin{array}{l}\text { Stamilio et al., } \\
2014 \text { [29] }\end{array}$ & 2008-2010 & USA & $\begin{array}{l}\text { Retrospective } \\
\text { cohort study }\end{array}$ & 585 & $\begin{array}{c}\text { - Emergency surgery } \\
\text { - Human } \\
\text { immunodeficiency } \\
\text { virus infection, } \\
\text { - Chronic corticosteroid } \\
\text { therapy or other } \\
\text { immunosuppressive } \\
\text { therapy, } \\
\text { - General anesthesia, } \\
\text { - Diagnosis of } \\
\text { extrauterine infection }\end{array}$ & $\begin{array}{c}\mathrm{BMI} \geq 30 \mathrm{~kg} / \mathrm{m}^{2} \\
\text { at delivery }\end{array}$ & $\begin{array}{l}\text { Composite of wound } \\
\text { infection and } \\
\text { endometritis }\end{array}$ \\
\hline $\begin{array}{l}\text { Conner et al., } \\
2014 \text { [10] }\end{array}$ & 2004-2008 & USA & $\begin{array}{l}\text { Retrospective } \\
\text { cohort study }\end{array}$ & 2444 & $\begin{array}{l}\text { - No completed follow- } \\
\text { up data }\end{array}$ & $\begin{array}{c}\mathrm{BMI} \geq 30 \mathrm{~kg} / \mathrm{m}^{2} \text { at } \\
\text { delivery }\end{array}$ & $\begin{array}{l}\text { A wound complication } \\
\text { defined as the } \\
\text { occurrence of a wound } \\
\text { seroma, hematoma, } \\
\text { separation, dehiscence } \\
\text { or infection from the } \\
\text { time of delivery to } \\
6 \text { weeks postoperative }\end{array}$ \\
\hline $\begin{array}{l}\text { Ibrahim et al., } \\
2014[30]\end{array}$ & $\begin{array}{l}\text { March 2012- } \\
\text { August } 2012\end{array}$ & Egypt & $\begin{array}{l}\text { Retrospective } \\
\text { cohort study }\end{array}$ & 130 & $\begin{array}{c}\text { - Infection (e.g., } \\
\text { chorioamnionitis, } \\
\text { pyelonephritis or chest } \\
\text { infection), intraoperative } \\
\text { events predispose to } \\
\text { perioperative infection } \\
\text { (e.g., bowel injury, } \\
\text { - Operative time more } \\
\text { than } 90 \text { min, } \\
\text { - Major blood loss } \\
\text { (hemoglobin less than } \\
10 \mathrm{~g} / \mathrm{dL} \text { ), } \\
\text { - Pre-eclampsia, diabetes } \\
\text { mellitus or rupture of } \\
\text { membranes for more } \\
\text { than } 12 \mathrm{~h}, \\
\text { - Immunosuppres- } \\
\text { sive drugs, } \\
\text { - non-Pfannenstiel incision, } \\
\text { - nonelective caesarean } \\
\text { section, } \\
\text { - BMI }<30 \mathrm{~kg} / \mathrm{m}^{2}\end{array}$ & $\begin{array}{c}\mathrm{BMI} \geq 30 \mathrm{~kg} / \mathrm{m}^{2} \\
\text { at delivery }\end{array}$ & $\begin{array}{l}\text { Superficial incisional } \\
\text { surgical site } \\
\text { infection (SSI) }\end{array}$ \\
\hline
\end{tabular}


Table 1. Cont.

\begin{tabular}{|c|c|c|c|c|c|c|c|}
\hline Study & Date & Country & Study Design & $N$ & Exclusion Criteria & Definition of Obesity & Primary Outcomes \\
\hline $\begin{array}{l}\text { Smid et al., } \\
2015 \text { [31] }\end{array}$ & 1999-2002 & USA & $\begin{array}{l}\text { Retrospective cohort } \\
\text { study }\end{array}$ & 38,299 & $\begin{array}{l}\text { - Incomplete demographic, } \\
\text { exposure, or outcome data }\end{array}$ & $\begin{array}{c}\mathrm{BMI} \geq 30-45 \mathrm{~kg} / \mathrm{m}^{2} \\
\text { extremely obese } \\
\mathrm{BMI} \geq 45 \mathrm{~kg} / \mathrm{m}^{2} \\
\text { at delivery }\end{array}$ & $\begin{array}{l}\text { A wound complication } \\
\text { composite of a wound } \\
\text { infection, endometritis, } \\
\text { a wound opening, a } \\
\text { seroma/hematoma, and } \\
\text { hospital readmission }\end{array}$ \\
\hline $\begin{array}{l}\text { Khalifa et al., } \\
2015 *[32]\end{array}$ & 2012-2013 & Egypt & $\begin{array}{l}\text { Randomized controlled } \\
\text { trial }\end{array}$ & 169 & $\begin{array}{l}\text { - Major intraoperative } \\
\text { complications (bowel or } \\
\text { urinary tract injuries, } \\
\text { massive blood loss, } \\
\text { transfusion) }\end{array}$ & $\mathrm{BMI} \geq 30 \mathrm{~kg} / \mathrm{m}^{2}$ & $\begin{array}{l}\text { The rate of superficial } \\
\text { surgical site infection }\end{array}$ \\
\hline $\begin{array}{l}\text { Sutton et al., } \\
2016 \text { [33] }\end{array}$ & 2010-2013 & USA & $\begin{array}{l}\text { Retrospective cohort } \\
\text { study }\end{array}$ & 421 & $\begin{array}{l}\text { - Prenatally diagnosed } \\
\text { fetal anomalies, } \\
\text { - Planned caesarean } \\
\text { hysterectomies }\end{array}$ & $\begin{array}{l}\mathrm{BMI} \geq 40 \mathrm{~kg} / \mathrm{m}^{2} \text { at the } \\
\text { time of delivery }\end{array}$ & $\begin{array}{l}\text { A wound composite } \\
\text { (cellulitis, abscess, } \\
\text { hematoma, seroma, } \\
\text { or dehiscence) }\end{array}$ \\
\hline $\begin{array}{l}\text { Zaki et al., } \\
2016 \text { [34] }\end{array}$ & 2006-2011 & USA & $\begin{array}{l}\text { Retrospective cohort } \\
\text { study }\end{array}$ & 1147 & $\begin{array}{c}\text { - Women with fascial } \\
\text { dehiscence, } \\
\text { - Multipregnancy, } \\
\text { - Second pregnancy }\end{array}$ & $\begin{array}{c}\text { Pre pregnancy } \\
\text { BMI } \geq 30 \mathrm{~kg} / \mathrm{m}^{2}\end{array}$ & $\begin{array}{l}\text { A composite of wound } \\
\text { disruption or infection } \\
\text { occurring within } \\
6 \text { weeks postpartum }\end{array}$ \\
\hline $\begin{array}{l}\text { Bindal \& Munda, } \\
\quad 2017^{*}[35]\end{array}$ & 2015-2016 & India & $\begin{array}{l}\text { Retrospective cohort } \\
\text { study }\end{array}$ & 100 & $\begin{array}{l}\text { - Major intraoperative } \\
\text { complications (bowel or } \\
\text { urinary tract injuries, } \\
\text { massive blood loss, } \\
\text { and transfusion) }\end{array}$ & $\begin{array}{c}\mathrm{BMI} \geq 30 \mathrm{~kg} / \mathrm{m}^{2} \text { at the } \\
\text { time of } \\
\text { caesarean section }\end{array}$ & $\begin{array}{l}\text { The rate of superficial } \\
\text { surgical site infection } \\
\text { defined as the presence } \\
\text { of wound discharge } \\
\text { that yielded a positive } \\
\text { result on } \\
\text { bacteriological culture }\end{array}$ \\
\hline $\begin{array}{l}\text { Looby et al., } \\
2017^{* *}[36]\end{array}$ & 2007-2014 & USA & $\begin{array}{l}\text { Retrospective cohort } \\
\text { study }\end{array}$ & 467 & $\begin{array}{l}\text { - Vaginal delivery } \\
\text { - BMI less than } 40 \mathrm{~kg} / \mathrm{m}^{2} \text {, } \\
\text { - Loss of follow-up, } \\
\text { - Multiple deliveries } \\
\text { - Use of nonpunch } \\
\text { through (NPT) devices } \\
\text { prior to official start date }\end{array}$ & $\mathrm{BMI} \geq 40 \mathrm{~kg} / \mathrm{m}^{2}$ & $\begin{array}{c}\text { A surgical site infection } \\
\text { (SSI), within } 30 \text { days } \\
\text { of surgery }\end{array}$ \\
\hline $\begin{array}{l}\text { Rusthaller et al., } \\
2017^{* *}[37]\end{array}$ & 2014-2016 & USA & $\begin{array}{l}\text { Retrospective cohort } \\
\text { study }\end{array}$ & 136 & $\begin{array}{c}\text { - Initiation of prenatal care } \\
\text { after } 23 \text { weeks (no early } \\
\text { BMI), } \\
\text { - Chronic steroid use, } \\
\text { - Pregestational diabetes, } \\
\text { treatment for an active } \\
\text { malignancy, } \\
\text { - Allergy to silver } \\
\text { (contraindication to } \\
\text { Prevena negative pressure } \\
\text { wound therapy device } \\
\text { (NPWT), } \\
\text { - Scheduled caesarean } \\
\text { section, } \\
\text { - Planned vertical } \\
\text { skin incision }\end{array}$ & $\begin{array}{c}\mathrm{BMI} \geq 30 \mathrm{~kg} / \mathrm{m}^{2} \text { at the } \\
\text { first prenatal visit } \\
<22 \text { weeks }\end{array}$ & $\begin{array}{l}\text { A composite of wound } \\
\text { morbidity at } 4 \text { weeks } \\
\text { postpartum including a } \\
\text { surgical site infection } \\
\text { (SSI) and/or } \\
\text { wound opening }\end{array}$ \\
\hline $\begin{array}{l}\text { Temming et al., } \\
2017 \text { [11] }\end{array}$ & 2011-2015 & USA & $\begin{array}{l}\text { Retrospective cohort } \\
\text { study }\end{array}$ & 1082 & $\begin{array}{l}\text { - Allergy to chlorhexidine, } \\
\text { alcohol, iodine or shellfish, } \\
\text { - Skin infection near the } \\
\text { operative site, } \\
\text { - Without follow-up } \\
\text { after discharge }\end{array}$ & $\mathrm{BMI} \geq 30 \mathrm{~kg} / \mathrm{m}^{2}$ & $\begin{array}{l}\text { A composite of wound } \\
\text { complications, } \\
\text { including surgical site } \\
\text { infection (SSI), cellulitis, } \\
\text { seroma, hematoma, and } \\
\text { separation within } \\
30 \text { days }\end{array}$ \\
\hline $\begin{array}{l}\text { Searle et al., } 2017 \\
* *[38]\end{array}$ & 2012-2016 & $\begin{array}{l}\text { Ireland, } \\
\text { England }\end{array}$ & $\begin{array}{l}\text { Retrospective cohort } \\
\text { study }\end{array}$ & 399 & $\begin{array}{l}\text { - Patient BMI }<35 \mathrm{~kg} / \mathrm{m}^{2} \\
\text { or patient BMI missing; } \\
\text { - Missing data in follow-up } \\
\text { time (time between } \\
\text { procedure date and } \\
\text { follow-up date) less than } \\
\text { seven days or missing }\end{array}$ & $\begin{array}{l}\mathrm{BMI} \geq 35 \mathrm{~kg} / \mathrm{m}^{2} \\
\quad \text { at the time of } \\
\text { caesarean section }\end{array}$ & $\begin{array}{l}\text { Postoperative wound } \\
\text { complications including } \\
\text { surgical site } \\
\text { infection (SSI) }\end{array}$ \\
\hline
\end{tabular}

* Studies evaluate subcutaneous drainage in obese women after a caesarean section. ${ }^{* *}$ Studies evaluate prophylactic negative pressure wound therapy in obese women after a caesarean section. 
Table 2. Characteristics of studies evaluating prophylactic antibiotics included in the systematic review.

\begin{tabular}{|c|c|c|c|c|c|c|c|}
\hline Study & Country & Study Design & $\mathrm{Pa}$ & $\begin{array}{l}\text { BMI of } \\
\text { articipants }\end{array}$ & Primary Outcomes & $\begin{array}{c}\text { Dose of } \\
\text { Antibiotics }\end{array}$ & Results \\
\hline $\begin{array}{l}\text { Wihbey et al., } \\
2018^{* *}[39]\end{array}$ & 2015-2017 & USA & $\begin{array}{l}\text { Retrospective } \\
\text { co- } \\
\text { hort } \\
\text { study }\end{array}$ & 166 & $\begin{array}{l}\text { - Under } 18 \text { years old, } \\
\text { - Did not speak } \\
\text { English, } \\
\text { - Had an allergy to } \\
\text { silver or } \\
\text { adhesive products, } \\
\text { - Skin incision that } \\
\text { would not fit the } \\
\text { device or standard } \\
\text { dressing (e.g., "T" } \\
\text { skin incision) }\end{array}$ & $\mathrm{BMI} \geq 35 \mathrm{~kg} / \mathrm{m}^{2}$ & $\begin{array}{l}\text { A superficial surgical site } \\
\text { infection, an infection } \\
\text { involving only the skin or } \\
\text { subcutaneous tissue } \\
\text { occurring within } 30 \text { days } \\
\text { of surgery with at least } \\
\text { one of the following: } \\
\text { purulent drainage from } \\
\text { the wound or organism } \\
\text { identified by culture or } \\
\text { wound deliberately } \\
\text { opened by the surgeon }\end{array}$ \\
\hline $\begin{array}{c}\text { Zaki et al., } 2018 \\
\text { [40] }\end{array}$ & 205-2016 & USA & $\begin{array}{l}\text { Retrospective } \\
\text { co- } \\
\text { hort } \\
\text { study }\end{array}$ & 242 & $\begin{array}{c}\text { - Hypersensitivity to } \\
\text { staples, } \\
\text { - Potential } \\
\text { immunosuppression } \\
\text { including infection } \\
\text { with human } \\
\text { immunodeficiency } \\
\text { virus, chronic steroid } \\
\text { use or active lupus }\end{array}$ & $\mathrm{BMI} \geq 40 \mathrm{~kg} / \mathrm{m}^{2}$ & $\begin{array}{l}\text { A composite wound } \\
\text { complication defined as a } \\
\text { superficial or deep } \\
\text { separation and infection } \\
\text { occurring up to } 6 \text { weeks } \\
\text { following delivery }\end{array}$ \\
\hline $\begin{array}{l}\text { Alalfy et al., } 2018 \\
\text { [41] }\end{array}$ & $\begin{array}{c}\text { From } \\
\text { June 2017- } \\
\text { December 2017 }\end{array}$ & Egypt & $\begin{array}{l}\text { Retrospective } \\
\text { co- } \\
\text { hort } \\
\text { study }\end{array}$ & 397 & $\begin{array}{c}\text { - BMI <30 kg } / \mathrm{m}^{2} \\
\text { - Previous caesarean } \\
\text { section } \\
\text { - Medical disorders } \\
\text { diabetes mellitus, } \\
\text { hypertension with } \\
\text { pregnancy }\end{array}$ & $\mathrm{BMI} \geq 30 \mathrm{~kg} / \mathrm{m}^{2}$ & $\begin{array}{l}\text { Wound outcome results } \\
\text { regarding postoperative } \\
\text { wound complications } \\
\text { compared to two widely } \\
\text { implemented techniques } \\
\text { in subcutaneous tissue } \\
\text { closure (interrupted } \\
\text { versus } \\
\text { continuous methods) }\end{array}$ \\
\hline $\begin{array}{c}\text { Marrs et al., } 2019 \\
\text { [42] }\end{array}$ & 2013-2017 & USA & $\begin{array}{l}\text { Retrospective } \\
\text { co- } \\
\text { hort } \\
\text { study }\end{array}$ & 91 & $\begin{array}{c}\text { - Rupture of } \\
\text { membranes for more } \\
\text { than } 18 \mathrm{~h}, \\
\text { - Clinical } \\
\text { chorioamnionitis at } \\
\text { the time of delivery, } \\
\text { - Subsequent vaginal } \\
\text { delivery, } \\
\text { - Participants enrolled } \\
\text { in other trials, } \\
\text { - Women with strong } \\
\text { indications for a } \\
\text { certain skin incision } \\
\text { type (i.e., placenta } \\
\text { accreta necessitating } \\
\text { caesarean } \\
\text { hysterectomy) }\end{array}$ & $\mathrm{BMI} \geq 40 \mathrm{~kg} / \mathrm{m}^{2}$ & $\begin{array}{l}\text { A composite wound } \\
\text { complication that } \\
\text { included any of the } \\
\text { following: surgical site } \\
\text { infection (SSI), cellulitis, } \\
\text { seroma/hematoma, or } \\
\text { separation up to } 6 \text { weeks } \\
\text { postpartum }\end{array}$ \\
\hline $\begin{array}{l}\text { Hussamy et al., } \\
2019 * *[43]\end{array}$ & 2015-2016 & USA & $\begin{array}{l}\text { Retrospective } \\
\text { co- } \\
\text { hort } \\
\text { study }\end{array}$ & 441 & $\begin{array}{l}\text { - Anticoagulation } \\
\text { therapy, } \\
\text { - Human } \\
\text { immunodeficiency } \\
\text { virus infection, } \\
\text { - Silver or acrylic } \\
\text { allergy }\end{array}$ & $\begin{array}{l}\mathrm{BMI} \geq 40 \mathrm{~kg} / \mathrm{m}^{2} \\
\text { measured within } \\
2 \text { weeks of } \\
\text { admission } \\
\text { for delivery }\end{array}$ & $\begin{array}{l}\text { A wound complication } \\
\text { defined as a wound } \\
\text { disruption or wound } \\
\text { infection (including } \\
\text { cellulitis) }\end{array}$ \\
\hline $\begin{array}{l}\text { Hyldig et al., } \\
2019^{* *}[44]\end{array}$ & 2013-2016 & Denmark & $\begin{array}{l}\text { Retrospective } \\
\text { co- } \\
\text { hort } \\
\text { study }\end{array}$ & 876 & $\begin{array}{l}\text { - Vaginal delivery, } \\
\text { - Missing data }\end{array}$ & $\begin{array}{c}\text { Pre-pregnancy } \\
\mathrm{BMI} \geq 30 \mathrm{~kg} / \mathrm{m}^{2}\end{array}$ & $\begin{array}{l}\text { A surgical site infection } \\
\text { was defined as a surgical } \\
\text { site infection requiring } \\
\text { antibiotic treatment } \\
\text { within the first } 30 \text { days } \\
\text { after the caesarean section }\end{array}$ \\
\hline $\begin{array}{l}\text { Connery et al., } \\
2019 \text { [45] }\end{array}$ & 2013-2016 & USA & $\begin{array}{l}\text { Retrospective } \\
\text { co- } \\
\text { hort } \\
\text { study }\end{array}$ & 657 & $\begin{array}{l}\text { - Patients did not } \\
\text { receive routine } \\
\text { prophylactic dose of } \\
\text { antibiotics in the } \\
\text { operating room, } \\
\text { - Skin incisions other } \\
\text { than Pfannenstiel, } \\
\text { - Uterine incisions } \\
\text { other than } \\
\text { low transverse, } \\
\text { - Patients with known } \\
\text { or discovered } \\
\text { allergies to silver or } \\
\text { nylon }\end{array}$ & $\mathrm{BMI} \geq 40 \mathrm{~kg} / \mathrm{m}^{2}$ & $\begin{array}{l}\text { A superficial surgical site } \\
\text { infection at any time } \\
\text { within the first } 6 \text { weeks } \\
\text { after caesarean delivery }\end{array}$ \\
\hline
\end{tabular}


Table 2. Cont.

\begin{tabular}{|c|c|c|c|c|c|c|c|}
\hline Study & Country & Study Design & $N$ & $\begin{array}{c}\text { BMI of } \\
\text { Participants }\end{array}$ & Primary Outcomes & $\begin{array}{c}\text { Dose of } \\
\text { Antibiotics }\end{array}$ & Results \\
\hline $\begin{array}{c}\text { Dias et al., } 2019 \text { * } \\
\text { [46] }\end{array}$ & 2011-2015 & Scotland & $\begin{array}{l}\text { Retrosp } \\
\text { co- } \\
\text { hort } \\
\text { study }\end{array}$ & ctive & -missing data & $\mathrm{BMI}>40 \mathrm{~kg} / \mathrm{m}$ & $\begin{array}{l}\text { Maternal and surgical } \\
\text { predictors of surgical site } \\
\text { infection }\end{array}$ \\
\hline $\begin{array}{l}\text { Tuuli et al., } 2020 \\
\qquad * *[47]\end{array}$ & 2017-2019 & USA & $\begin{array}{l}\text { Retrosp } \\
\text { co- } \\
\text { hort stu }\end{array}$ & $\begin{array}{l}\text { ctive } \\
\text { y }\end{array}$ & $\begin{array}{c}\text { - Postoperative } \\
\text { follow-up } \\
\text { not available, } \\
\text { - Contraindication to } \\
\text { negative pressure use } \\
\text { (pre-existing infection } \\
\text { at the incision site), } \\
\text { - Bleeding disorder, } \\
\text { - Therapeutic } \\
\text { anticoagulation, } \\
\text { - Allergy to silicone or } \\
\text { adhesive tape }\end{array}$ & $\begin{array}{l}\mathrm{BMI} \geq 30 \mathrm{~kg} / \mathrm{m}^{2} \\
\text { at or beyond } \\
23 \text { weeks of } \\
\text { gestation }\end{array}$ & $\begin{array}{l}\text { A superficial or deep } \\
\text { surgical site infection }\end{array}$ \\
\hline $\begin{array}{l}\text { Pevzner et al., } \\
2011[48]\end{array}$ & USA & Prospective study & 29 & $\begin{array}{c}\mathrm{BMI}<30 \mathrm{~kg} / \mathrm{m}^{2} \\
(n=10) \\
\mathrm{BMI} 30-39.9 \\
\mathrm{~kg} / \mathrm{m}^{2}(n=10) \\
\mathrm{BMI} \geq \underset{(n=9)}{\geq 40 \mathrm{~kg} / \mathrm{m}^{2}}\end{array}$ & $\begin{array}{c}\text { Cefazolin } \\
\text { concentration in } \\
\text { adipose tissue and } \\
\text { surgical site infection }\end{array}$ & $\begin{array}{l}2 \mathrm{~g} \text { of cefazolin } \\
30-60 \text { min before } \\
\text { skin incision }\end{array}$ & $\begin{array}{l}\text { No significant difference } \\
\text { in cefazolin concentration } \\
\text { observed in mean closure } \\
\text { adipose, myometrial or } \\
\text { serum specimens across } \\
\text { the BMI categories }\end{array}$ \\
\hline $\begin{array}{l}\text { Stitely et al., } \\
2013 \text { [49] }\end{array}$ & New Zealand & $\begin{array}{l}\text { Retrospective } \\
\text { cohort study }\end{array}$ & 20 & $\mathrm{BMI} \geq 35 \mathrm{~kg} / \mathrm{m}^{2}$ & $\begin{array}{c}\text { Tissue concentration } \\
\text { of antibiotics }\end{array}$ & $\begin{array}{l}\text { Cefazolin } 2 \text { g vs. } \\
4 \text { g i.v. }\end{array}$ & $\begin{array}{l}\text { Mean cefazolin plasma, } \\
\text { umbilical cord, and } \\
\text { myometrial } \\
\text { concentrations } \\
\text { significantly higher in the } \\
4 \mathrm{~g} \text { treatment group } \\
(p<0.05)\end{array}$ \\
\hline $\begin{array}{c}\text { Ahmadzia et al., } \\
\text { 2015 [50] }\end{array}$ & USA & $\begin{array}{l}\text { Retrospective } \\
\text { cohort study }\end{array}$ & 335 & $>250$ pounds & $\begin{array}{c}\text { Incidence of surgical } \\
\text { site infections, } \\
\text { (superficial, deep, } \\
\text { and } \\
\text { organ/space-i.e., } \\
\text { endometritis } \\
\text { infections) }\end{array}$ & $\begin{array}{l}\text { Cefazolin } 2 \mathrm{~g} \text { vs. } \\
\text { cefazolin } 3 \mathrm{~g}\end{array}$ & $\begin{array}{l}\text { No difference in surgical } \\
\text { site infection among } \\
\text { women who received } 2 \mathrm{~g} \\
\text { compared with } \\
3 \mathrm{~g} \text { cefazolin }\end{array}$ \\
\hline $\begin{array}{l}\text { Young et al., } \\
2015 \text { [51] }\end{array}$ & USA & $\begin{array}{l}\text { Double-blind } \\
\text { randomized } \\
\text { controlled trial }\end{array}$ & 28 & $\begin{array}{c}\text { A pregnancy } \\
\mathrm{BMI} \geq 30 \mathrm{~kg} / \mathrm{m}^{2}\end{array}$ & $\begin{array}{l}\text { Cefazolin } \\
\text { Concentrations in } \\
\text { maternal plasma, } \\
\text { umbilical cord blood, } \\
\text { and maternal } \\
\text { adipose tissue }\end{array}$ & $\begin{array}{l}2 \mathrm{~g} \text { or } 3 \mathrm{~g} \\
\text { cefazolin } \\
\text { concentrations } \\
\text { within } 30 \text { min of } \\
\text { skin incision }\end{array}$ & $\begin{array}{l}\text { Cefazolin concentrations } \\
\text { in plasma and adipose } \\
\text { tissue were related to both } \\
\text { dose and body mass index. } \\
\text { No difference between } 2 \\
\text { and } 3 \text { g cefazolin doses to } \\
\text { maintain adipose tissue } \\
\text { concentrations above the } \\
\text { minimum } \\
\text { inhibitory concentration }\end{array}$ \\
\hline $\begin{array}{l}\text { Swank et al., } \\
2015 \text { [52] }\end{array}$ & USA & $\begin{array}{l}\text { Prospective } \\
\text { control study }\end{array}$ & 29 & $\mathrm{BMI} \geq 30 \mathrm{~kg} / \mathrm{m}^{2}$ & $\begin{array}{l}\text { Cefazolin } \\
\text { concentration } \\
\text { in tissue }\end{array}$ & $\begin{array}{c}2 \mathrm{~g} \text { or } 3 \mathrm{~g} \\
\text { cefazolin } \\
\text { concentrations } \\
\text { within } 30-60 \mathrm{~min} \\
\text { of skin incision }\end{array}$ & $\begin{array}{c}\text { Higher adipose } \\
\text { concentrations of cefazolin } \\
\text { were observed after the } \\
\text { administration of an } \\
\text { increased } \\
\text { prophylactic dose }\end{array}$ \\
\hline $\begin{array}{l}\text { Maggio et al., } \\
2015[53]\end{array}$ & USA & $\begin{array}{l}\text { Double-blind } \\
\text { randomized } \\
\text { controlled trial }\end{array}$ & 57 & $\mathrm{BMI} \geq 30 \mathrm{~kg} / \mathrm{m}^{2}$ & $\begin{array}{l}\text { Adipose tissue } \\
\text { cefazolin } \\
\text { concentration }\end{array}$ & $\begin{array}{c}2 \mathrm{~g} \text { vs. } \\
3 \mathrm{~g} \text { cefazolin }\end{array}$ & $\begin{array}{c}\text { Prophylaxis with } 3 \mathrm{~g} \text { of } \\
\text { cefazolin did not } \\
\text { significantly increase } \\
\text { adipose } \\
\text { tissue concentration }\end{array}$ \\
\hline $\begin{array}{l}\text { Lilico et al., } \\
2016 \text { [54] }\end{array}$ & Canada & $\begin{array}{l}\text { Prospective } \\
\text { control study }\end{array}$ & 6 & $\mathrm{BMI} \geq 35 \mathrm{~kg} / \mathrm{m}^{2}$ & $\begin{array}{l}\text { Cefazolin } \\
\text { concentration } \\
\text { in tissue }\end{array}$ & $\begin{array}{l}25 \mathrm{mg} / \mathrm{kg} \text { of } \\
\text { cefazolin }\end{array}$ & $\begin{array}{l}\text { Obese patients required a } \\
\text { higher dose of cefazolin }\end{array}$ \\
\hline $\begin{array}{l}\text { Groff et al., } \\
2017 \text { [55] }\end{array}$ & USA & $\begin{array}{l}\text { Prospective } \\
\text { controlled study }\end{array}$ & 8 & $\mathrm{BMI} \geq 30 \mathrm{~kg} / \mathrm{m}^{2}$ & $\begin{array}{l}\text { Prevention of } \\
\text { wound infection }\end{array}$ & $2 \mathrm{~g}$ of cefazolin & $\begin{array}{l}\text { No difference between } \\
\text { groups in total and free } \\
\text { cefazolin concentrations }\end{array}$ \\
\hline $\begin{array}{l}\text { Gupper et al., } \\
2017[56]\end{array}$ & USA & $\begin{array}{l}\text { Analysis of } 3 \\
\text { retrospective con- } \\
\text { trolled studies }\end{array}$ & 67 & $\mathrm{BMI} \geq 30 \mathrm{~kg} / \mathrm{m}^{2}$ & $\begin{array}{l}\text { Cefazolin adipose } \\
\text { tissue concentration }\end{array}$ & $\begin{array}{c}2 \mathrm{~g} \text { or } 3 \mathrm{~g} \\
\text { cefazolin } \\
\text { concentrations } \\
\text { within } 30-60 \mathrm{~min} \\
\text { of skin incision }\end{array}$ & $\begin{array}{l}\text { A } 2 \text { g dose of cefazolin } \\
\text { had a high probability of } \\
\text { providing adipose tissue } \\
\text { concentrations above the } \\
\text { target pathogens' MIC for } \\
\text { overweight and } \\
\text { obese females }\end{array}$ \\
\hline
\end{tabular}


Table 2. Cont.

\begin{tabular}{|c|c|c|c|c|c|c|c|}
\hline Study & Country & Study Design & $N$ & $\begin{array}{c}\text { BMI of } \\
\text { Participants }\end{array}$ & Primary Outcomes & $\begin{array}{c}\text { Dose of } \\
\text { Antibiotics }\end{array}$ & Results \\
\hline $\begin{array}{c}\text { Kram et al., } \\
2017 \text { [57] }\end{array}$ & USA & $\begin{array}{l}\text { Prospective } \\
\text { controlled study }\end{array}$ & 84 & $\mathrm{BMI} \geq 30 \mathrm{~kg} / \mathrm{m}^{2}$ & $\begin{array}{l}\text { Cefazolin blood and } \\
\text { adipose tissue } \\
\text { concentration }\end{array}$ & $\begin{array}{l}2 \mathrm{~g} \text { and } 3 \mathrm{~g} \text { of } \\
\text { cefazolin for } \\
\text { body weights } \\
<120 \mathrm{~kg} \text { and } \\
\geq 120 \mathrm{~kg}\end{array}$ & $\begin{array}{l}\text { Dosage groups did not } \\
\text { differ in cefazolin } \\
\text { concentration (median } \\
\text { [interquartile range]) in } \\
\text { adipose tissue following } \\
\text { skin incision, and in } \\
\text { adipose tissue before skin } \\
\text { closure. Mean } \\
\text { concentrations were } \\
\text { significantly lower than } \\
\text { the MIC of } 8 \mathrm{mg} / \mathrm{g} \\
(P<0.03) \text { in both groups }\end{array}$ \\
\hline $\begin{array}{l}\text { Eley et al., } \\
2020 \text { [58] }\end{array}$ & Australia & Prospective study & 12 & $\mathrm{BMI} \geq 35 \mathrm{~kg} / \mathrm{m}^{2}$ & $\begin{array}{c}\text { Plasma and } \\
\text { interstitial fluid } \\
\text { pharmacokinetics of } \\
\text { intravenous cefazolin }\end{array}$ & $\begin{array}{c}2 \mathrm{~g} \text { of } \\
\text { cefazolin i.v. }\end{array}$ & $\begin{array}{l}\text { Wound closure did not } \\
\text { occur within } 2 \mathrm{~h} \text {; redosing } \\
\text { is suggested following } \\
\text { either a } 2 \text { or } 3 \mathrm{~g} \\
\text { initial bolus }\end{array}$ \\
\hline
\end{tabular}

\subsection{Synthesis of Results}

\subsubsection{Relationship between Preoperative Factors and Wound Complications}

Hypertension, like diabetes, is a disease that often coexists with obesity. Seven randomized trials investigate the effect of these diseases on wound healing [11,24,26-28,34,40], and take into account the premature rupture of the membranes, concomitant infections of the membranes or smoking. An American retrospective study found that 1 in 3 women who are morbidly obese will develop complications in the surgical wound, and smoking increases the risk of these complications by more than double (RR 2.7 [95\% CI 1.08-6.54] $p=0.03$ ) [24]. Another study confirmed that smoking and longitudinal skin incisions significantly increase the risk of infections and wound dehiscence after a caesarean section [40]. However, Temming and colleagues, in a study involving 1082 patients with a BMI of $>30$ did not confirm that obesity, smoking, diabetes, and chorioamnionitis had a significant impact on the risk of complications in the postoperative wound if the surgery was performed according to evidence-based medicine, that is, prophylactic antibiotics administered up to $60 \mathrm{~min}$ before the skin incision, the skin washed with an alcohol solution of chlorhexidine, the subcutaneous tissue sutured if its thickness is greater than $2 \mathrm{~cm}$, and the skin sutured with an intradermal stitch. In this case, the only significant risk factor for wound disorders (27.5\% vs. $16.1 \%$, RR 1.71 [95\% CI 1.12-247]) and the occurrence of SSIs (6.9\% vs. $1.6 \%$, RR 3.74 [ $95 \%$ CI 1.18-11.92]) is the fact of an emergency caesarean section [11]. Other authors showed no significant influence of diabetes, hypertension, the premature outflow of amniotic fluid or inflammation of the membranes on the increased incidence of postoperative wound complications in obese women [24,26-28,34].

\subsubsection{Antibiotic Prophylaxis}

The estimation of the optimal prophylactic dose of an antibiotic reaching a concentration higher than the minimum inhibitory dose (minimum inhibitory concentration)-both in the blood and in the adipose tissue-reducing the risk of postoperative wound infection, remains the subject of many studies [48-58]. In a 2011 study, Pevzner and colleagues questioned the effectiveness of a prophylactic dose of $2 \mathrm{~g}$ of cefazolin in women with varying degrees of obesity [48]. This was confirmed by Swank and colleagues, who showed that the minimal inhibitory concentration (MIC) was not reached in adipose tissue in relation to Gram-negative bacteria at the time of skin incision in $20 \%$ of obese women (BMI $30-40 \mathrm{~kg} / \mathrm{m}^{2}$ ) and $44 \%$ of morbidly obese women after the use of cefazolin in a dose of $2 \mathrm{~g}$. Increasing the dose to $3 \mathrm{~g}$ resulted in all the obese and $71 \%$ of the morbidly obese women reaching MIC $\geq 8 \mu \mathrm{g} / \mathrm{mL}$ [52]. In a randomized Australian cohort study involving 2231 women, increasing the dose of cefazolin to $3 \mathrm{~g}$ in women with a BMI of $\geq 30 \mathrm{~kg} / \mathrm{m}^{2}$ resulted in a significant reduction in the occurrence of SSIs (OR 0.309, $p<0.001$ ) [59]. In a randomized, double-blind study conducted among pregnant women in labor with a 
BMI of above 30, increasing the dose of cefazolin to $3 \mathrm{~g}$ did not significantly increase its concentration in adipose tissue and did not show significantly greater protection against the Staphylococcus species compared to the group receiving a dose of $2 \mathrm{~g}(61 \% \mathrm{vs} .72 \%$, $p=0.35$ ) [53]. In another retrospective US cohort study involving 335 obese women, increasing the prophylactic dose also did not reduce the incidence of SSIs [50]. In a randomized, double-blind study, Young et al. showed that although the concentration of the antibiotic in both blood serum and pregnant adipose tissue is dependent on the dose of the drug and the body weight, the use of both $2 \mathrm{~g}$ and $3 \mathrm{~g}$ of cefazolin is the optimal protection in the ratio of Gram-positive and Gram-negative bacteria [51]. Similar conclusions were provided by the study conducted by Groff and colleagues, which showed that the intravenous administration of $2 \mathrm{~g}$ of cefazolin fully protects against postoperative wound infections in both obese and normal-weight women. Moreover, this dose in both groups protects the newborn against an infection with the Streptococcus group B (GBS) and S. aureus [55]. Another American study investigating the penetration of an antibiotic into adipose tissue showed that the administration of $2 \mathrm{~g}$ of cefazolin 30-60 min before the skin incision reached concentrations above the minimum inhibitory concentration in both overweight and obese women if the procedure lasts less than 90 min (the probability of target attainment (PTA) in adipose tissue for $2 \mathrm{~g}$ of cefazoline was $92.4 \%$, and $94.7 \%$ for $3 \mathrm{~g}$ of cefazoline). If the duration of the surgery exceeds $2 \mathrm{~h}$, the PTA for $2 \mathrm{~g}$ of cefazoline was $86.8 \%$, suggesting the need for another dose of antibiotic [56]. Eley et al. also confirmed that another prophylactic dose in the case of routine, uncomplicated patients is not needed [58]. However, Guper et al. showed that both the total body weight (TBW) and the BMI had no effect on the concentration of cefazolin in adipose tissue [56]. On the other hand, Kram and colleagues, in a study involving 84 patients with obesity of at least grade 1, found that its mean concentration in adipose tissue is still below the MIC of $8 \mathrm{mg} / \mathrm{g}(p<0.03)$ regardless of the antibiotic dose used [57]. The results of the analysis are presented in Table 3.

Table 3. Results of studies that included a prophylactic dose of cefazolin.

\begin{tabular}{|c|c|c|c|c|c|}
\hline Study & $N$ & BMI of Participants & Primary Outcomes & $\begin{array}{c}\text { Dose of } \\
\text { Antibiotics }\end{array}$ & Results \\
\hline Pevzner et al., [48] & 29 & $\begin{array}{c}\mathrm{BMI}<30 \mathrm{~kg} / \mathrm{m}^{2}(n=10) \\
\mathrm{BMI} 30-39.9 \mathrm{~kg} / \mathrm{m}^{2}(n=10) \\
\mathrm{BMI} \geq 40 \mathrm{~kg} / \mathrm{m}^{2}(n=9)\end{array}$ & $\begin{array}{l}\text { Cefazolin concentration in } \\
\text { adipose tissue and surgical } \\
\text { site infection }\end{array}$ & $\begin{array}{l}2 \mathrm{~g} \text { of cefazolin } 30-60 \\
\text { min before the } \\
\text { skin incision }\end{array}$ & $\begin{array}{l}\text { No significant difference in } \\
\text { cefazolin concentration was } \\
\text { observed in mean closure } \\
\text { adipose, myometrial, or serum } \\
\text { specimens across the } \\
\text { BMI categories }\end{array}$ \\
\hline Stitely et al., [49] & 20 & $\mathrm{BMI} \geq 35 \mathrm{~kg} / \mathrm{m}^{2}$ & $\begin{array}{c}\text { Tissue concentration } \\
\text { of antibiotics }\end{array}$ & Cefazolin 2 g vs. 4 g i.v. & $\begin{array}{l}\text { The mean cefazolin plasma, } \\
\text { umbilical cord, and myometrial } \\
\text { concentrations were significantly } \\
\text { higher in the } 4 \text { g treatment group } \\
\qquad(p<0.05)\end{array}$ \\
\hline Ahmadzia et al., [50] & 335 & $>250$ pounds & $\begin{array}{l}\text { Incidence of surgical site } \\
\text { infections, (superficial, deep, } \\
\text { and organ/space-i.e., } \\
\text { endometritis) infections) }\end{array}$ & $\begin{array}{l}\text { Cefazolin } 2 \mathrm{~g} \text { vs. } \\
\text { cefazolin } 3 \mathrm{~g}\end{array}$ & $\begin{array}{l}\text { No difference in surgical site } \\
\text { infection among women who } \\
\text { received } 2 \text { g compared with } 3 \mathrm{~g} \\
\text { cefazolin }\end{array}$ \\
\hline Young et al., [51] & 28 & Pregnancy BMI $\geq 30 \mathrm{~kg} / \mathrm{m}^{2}$ & $\begin{array}{c}\text { Cefazolin concentrations in } \\
\text { maternal plasma, umbilical } \\
\text { cord blood, and maternal } \\
\text { adipose tissue }\end{array}$ & $\begin{array}{l}2 \mathrm{~g} \text { or } 3 \mathrm{~g} \text { within } 30 \mathrm{~min} \\
\text { of the skin incision }\end{array}$ & $\begin{array}{l}\text { Cefazolin concentrations in } \\
\text { plasma and adipose tissue are } \\
\text { related to both the dose and the } \\
\text { body mass index. No difference } \\
\text { between } 2 \mathrm{~g} \text { and } 3 \mathrm{~g} \text { cefazolin } \\
\text { doses to maintain adipose tissue } \\
\text { concentrations above the } \\
\text { minimum } \\
\text { inhibitory concentration }\end{array}$ \\
\hline Swank et al., [52] & 29 & $\mathrm{BMI} \geq 30 \mathrm{~kg} / \mathrm{m}^{2}$ & $\begin{array}{l}\text { Cefazolin concentration } \\
\text { in tissue }\end{array}$ & $\begin{array}{l}2 \mathrm{~g} \text { or } 3 \mathrm{~g} \text { within } 30-60 \\
\text { min of the skin incision }\end{array}$ & $\begin{array}{l}\text { Higher adipose concentrations of } \\
\text { cefazolin were observed after the } \\
\text { administration of an increased } \\
\text { prophylactic dose }\end{array}$ \\
\hline Maggio et al., [53] & 57 & $\mathrm{BMI} \geq 30 \mathrm{~kg} / \mathrm{m}^{2}$ & $\begin{array}{l}\text { Adipose tissue cefazolin } \\
\text { concentration measured by } \\
\text { high pressure liquid } \\
\text { chromatography }\end{array}$ & 2 g vs. 3 g cefazolin & $\begin{array}{l}\text { Prophylaxis with } 3 \mathrm{~g} \text { of cefazolin } \\
\text { did not significantly increase } \\
\text { adipose tissue concentration }\end{array}$ \\
\hline
\end{tabular}


Table 3. Cont.

\begin{tabular}{|c|c|c|c|c|c|}
\hline Study & $N$ & BMI of Participants & Primary Outcomes & $\begin{array}{c}\text { Dose of } \\
\text { Antibiotics }\end{array}$ & Results \\
\hline Lilico et al., [54] & 6 & $\mathrm{BMI} \geq 35 \mathrm{~kg} / \mathrm{m}^{2}$ & $\begin{array}{l}\text { Cefazolin concentration } \\
\text { in tissue }\end{array}$ & $25 \mathrm{mg} / \mathrm{kg}$ of cefazolin & $\begin{array}{l}\text { Obese patients need a higher } \\
\text { dose of cefazolin }\end{array}$ \\
\hline Groff et al., [55] & 8 & $\mathrm{BMI} \geq 30 \mathrm{~kg} / \mathrm{m}^{2}$ & $\begin{array}{c}\text { Prevention of } \\
\text { wound infection }\end{array}$ & $2 \mathrm{~g}$ of cefazolin & $\begin{array}{c}\text { No difference between groups in } \\
\text { total and free } \\
\text { cefazolin concentrations }\end{array}$ \\
\hline Gupper et al., [56] & 67 & $\mathrm{BMI} \geq 30 \mathrm{~kg} / \mathrm{m}^{2}$ & $\begin{array}{l}\text { Cefazolin adipose } \\
\text { tissue concentration }\end{array}$ & $\begin{array}{l}2 \mathrm{~g} \text { or } 3 \mathrm{~g} \text { within } 30-60 \\
\text { min of skin incision }\end{array}$ & $\begin{array}{l}2 \mathrm{~g} \text { dose has a high probability of } \\
\text { providing adipose tissue } \\
\text { concentrations above the target } \\
\text { pathogens' MIC for overweight } \\
\text { and obese females }\end{array}$ \\
\hline Kram et al., [57] & 84 & $\mathrm{BMI} \geq 30 \mathrm{~kg} / \mathrm{m}^{2}$ & $\begin{array}{l}\text { Cefazolin blood and adipose } \\
\text { tissue concentration }\end{array}$ & $\begin{array}{l}2 \mathrm{~g} \text { and } 3 \mathrm{~g} \text { of cefazolin } \\
\text { for body weights of } \\
<120 \mathrm{~kg} \text { and } \geq 120 \mathrm{~kg}\end{array}$ & $\begin{array}{l}\text { Dosage groups did not differ in } \\
\text { cefazolin concentration (median } \\
\text { [interquartile range]) in adipose } \\
\text { tissue following the skin incision, } \\
\text { and in adipose tissue before the } \\
\text { skin closure. Mean } \\
\text { concentrations were significantly } \\
\text { lower than the MIC of } 8 \mathrm{mg} / \mathrm{g} \\
(P<0.03) \text { in both groups }\end{array}$ \\
\hline Eley et al., [58] & 12 & $\mathrm{BMI} \geq 35 \mathrm{~kg} / \mathrm{m}^{2}$ & $\begin{array}{c}\text { plasma and interstitial fluid } \\
\text { pharmacokinetics of } \\
\text { intravenous cefazolin }\end{array}$ & $2 \mathrm{~g}$ of cefazolin i.v. & $\begin{array}{l}\text { Wound closure did not occur } \\
\text { within } 2 \mathrm{~h} \text {; redosing is suggested } \\
\text { following either a } 2 \text { or } 3 \mathrm{~g} \\
\text { initial bolus }\end{array}$ \\
\hline
\end{tabular}

BMI—body mass index.

\subsubsection{Skin Incisions}

An incision of the skin and related complications within the postoperative wound were the subject of six retrospective randomized studies in which the incidence of complications following a transverse or vertical skin incision was analyzed. Most authors take into account the risk of wound infection and its dehiscence, as well as the presence of fluid collections (seroma and hematoma) resulting in rehospitalization. In a US cohort study of morbidly obese pregnant women, the risk of postoperative wound complications following a vertical abdominal incision more than doubled (OR 2.2 (1.18-4.27)) [24]. Another study involving 242 women with a BMI of $\geq 30 \mathrm{~kg} / \mathrm{m}^{2}$ indicated that obesity significantly increases the risk of complications within a postoperative wound, although the method of skin incision had no effect on the incidence of this complication [26]. Smid et al., in an analysis of 2411 pregnant women, showed that those with morbid obesity $\left(\mathrm{BMI}>45 \mathrm{~kg} / \mathrm{m}^{2}\right.$ ) are at increased risk of endometritis (AOR 1.26; 95\% CI 1.07-1.49) and wound infections (AOR 3.77; 95\% CI 2.60-5.46) compared to women with normal body weight. Moreover, he showed that infections accompany vertical incisions of the skin significantly more often $(p=0.02)$ than transverse incisions [31]. Thornburg et al. assessed that vertical incisions increase the risk of wound complications by more than seven-fold. There is an increased risk of infection (OR 5.16; 95\% CI 2.3-11.8) and wound dehiscence (OR 10.7; 95\% CI 4.0-29.2) in obese women, regardless of the degree of their obesity [27]. In the study from US, the authors showed a significantly lower incidence of complications within the wound (including infection, seroma, hematoma, and fascial dehiscence) in the case of vertical incisions of the skin (OR 0.32; 95\% CI 0.17-0.62) [42]. The results are presented in Table 4. 
Table 4. Types of skin incisions and wound complications.

\begin{tabular}{|c|c|c|c|c|c|}
\hline \multirow{2}{*}{ Study } & \multirow{2}{*}{$N$} & \multirow{2}{*}{ BMI $\left(\mathrm{kg} / \mathrm{m}^{2}\right)$} & \multicolumn{2}{|c|}{ Wound Complications } & \multirow{2}{*}{ Results } \\
\hline & & & $N$ & OR & \\
\hline Alanis et al. [24] & 194 & $\geq 50$ & $\begin{array}{l}\text { V } 39 / 104 \\
\text { T } 19 / 90\end{array}$ & $2.24(1.18-4.27)$ & $\begin{array}{c}\text { Vertical abdominal incisions were } \\
\text { associated with } \\
\text { wound complications }\end{array}$ \\
\hline McLean et al. [26] & 242 & $\geq 30$ & $\begin{array}{l}\mathrm{V} 5 / 25 \\
\mathrm{~T} 22 / 213\end{array}$ & $2.17(0.74-6.36)$ & $\begin{array}{l}\text { Type of skin incision does not } \\
\text { appear to be associated with wound } \\
\text { complications in obese patients }\end{array}$ \\
\hline Tornburg et al. [27] & 623 & $\geq 35$ & $\begin{array}{l}\text { V } 16 / 35 \\
\text { T } 68 / 588\end{array}$ & $6.44(3.16-13.12)$ & $\begin{array}{c}\text { Vertical skin incisions are } \\
\text { associated with an increased risk of } \\
\text { wound complications }\end{array}$ \\
\hline Smid et al. [31] & 2411 & $>45$ & $\begin{array}{l}\text { V } 113 / 669 \\
\text { T } 17 / 224\end{array}$ & $2.47(1.45-4.22)$ & $\begin{array}{l}\text { Vertical skin incisions are } \\
\text { associated with an increased risk of } \\
\text { wound complications }\end{array}$ \\
\hline Sutton et al. [33] & 421 & $\geq 40$ & $\begin{array}{l}\text { V } 15 / 57 \\
\text { T } 54 / 364\end{array}$ & $2.05(1.06-3.95)$ & $\begin{array}{l}\text { Vertical incisions are associated } \\
\text { with more wound complications }\end{array}$ \\
\hline Marrs et al. [42] & 91 & $\geq 40$ & $\begin{array}{l}\text { V } 8 / 38 \\
\text { T } 8 / 43\end{array}$ & $1.17(0.39-3.49)$ & $\begin{array}{l}\text { Pfannenstiel skin incisions were } \\
\text { associated with lower } \\
\text { wound complications }\end{array}$ \\
\hline
\end{tabular}

V—vertical skin incision, $\mathrm{T}$ — transverse skin incision, $\mathrm{N}$-number of cases, $\mathrm{OR}$-odds ratio, $\mathrm{BMI}$-body mass index.

The impact of the type of skin incision on the risk of wound complication in obese women was assessed based on six studies with a total number of 928 women with vertical skin incision and 1522 obese women with transverse skin incision. Pooling the data together, we observed that wound complications were present in $21 \%$ of obese women with vertical incision but only in $12 \%$ of obese women with transverse incision. Studies were mildly moderately heterogeneous ( $\mathrm{I}^{2} 46 \%$ with $p=0.10$ ) thus the FEM method was used to calculate the pooled OR. Wound complications were found to be significantly more frequent in women with vertical skin incision than in women with transverse skin incision ( $21 \%$ vs. $12 \%)$. The value of pooled OR equals to 2.48 (95\% CI $1.85-3.32, p<0.01)$ indicates that the odd of wound complication in obese women after a caesarean section is almost 2.5 -fold higher in the case of vertical skin incisions (Figure 2). No publication bias was found for this analysis [Egger's test $(p=0.633)$; Begg's test $(p=0.348)]$ and sensitivity analysis demonstrated that the results were stable and reliable.

In addition, we performed subgroup analysis based on four studies considering women with BMI $\geq 40 \mathrm{~kg} / \mathrm{m}^{2}$, including 868 women with vertical skin incision and 721 women with transverse skin incision in total. There was no heterogeneity between the studies $\left(\mathrm{I}^{2} 0 \%\right)$. The analysis revealed that wound complications were again significantly more frequent in women with extreme obesity and vertical skin incision than in extremely obese women with transverse skin incision ( $20 \%$ vs. $14 \%$, respectively). The results showed that the odd of wound complication in women with BMI $\geq 40 \mathrm{~kg} / \mathrm{m}^{2}$ after caesarean section is almost 2.2-fold higher in the case of vertical skin incision than transverse skin incision $(\mathrm{OR}=2.17(95 \% \mathrm{CI} 1.56-3.03, p<0.01)$ (Figure 2). However, in this analysis publication bias was observed [Egger's test $(p=0.008)$; Begg's test $(p=0.041)$ ]. In turn, sensitivity analysis showed that the results of OR calculation were stable. 


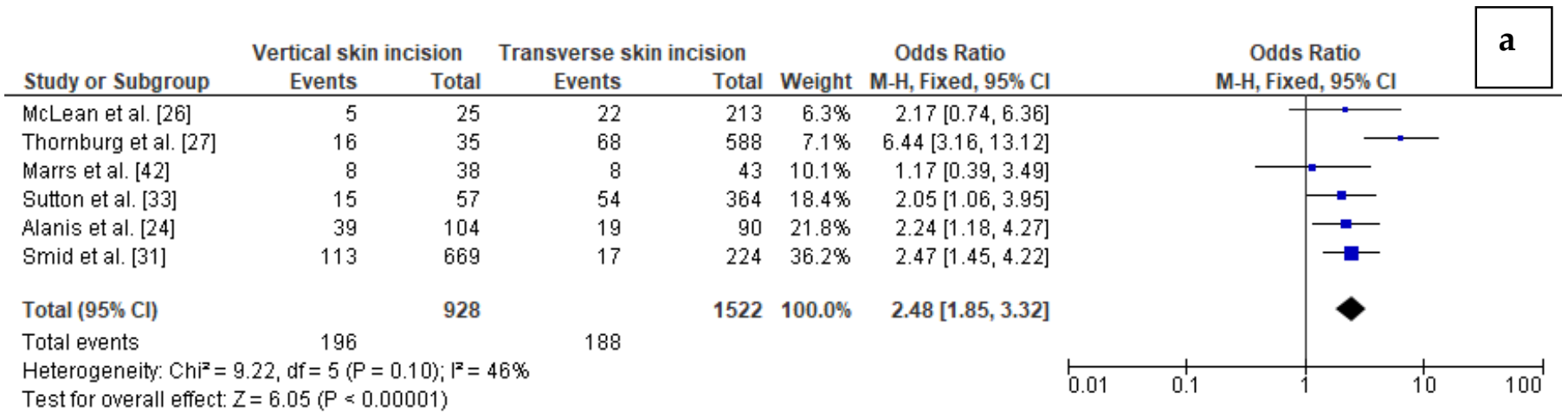

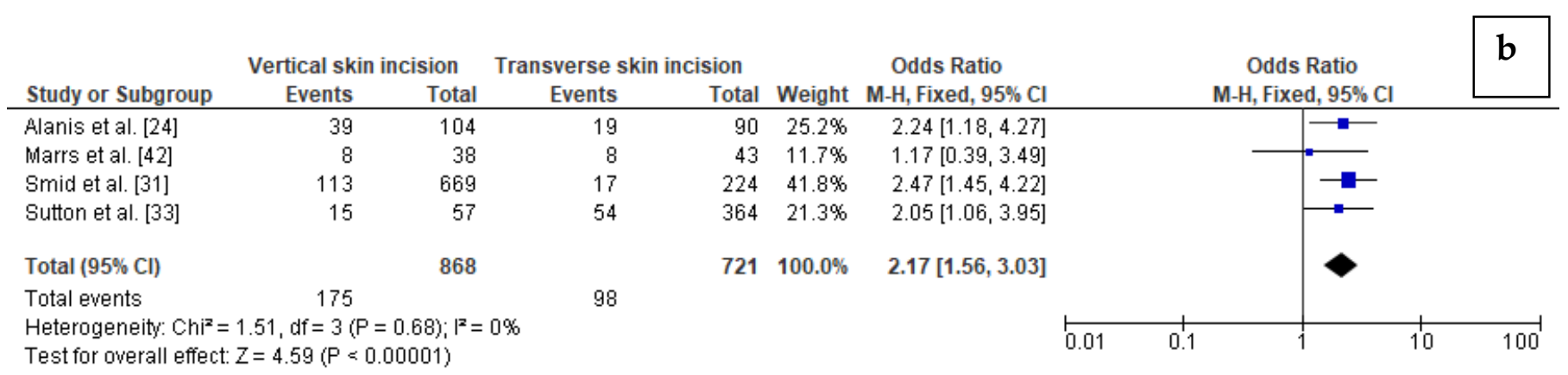

Figure 2. Forest plots for: (a) wound complications in the group of post-caesarean obese women with vertical skin incision and in women with transverse skin incision; (b) wound complications in the group of post-caesarean women with $\mathrm{BMI} \geq 40 \mathrm{~kg} / \mathrm{m}^{2}$ and vertical skin incision compared to women with transverse skin incision. M.-H.-Mantel-Haenszel; $\mathrm{CI}$-confidence interval; $\mathrm{I}^{2}$ — heterogeneity; $\mathrm{df}$ - degrees of freedom.

\subsubsection{Skin and Subcutaneous Tissue Closure}

Four randomized trials compared the effectiveness of stitching skin with staplers, single sutures, and intradermal sutures. An Egyptian study conducted among 130 women diagnosed with obesity whose skin was closed with an intradermal suture showed a significantly higher risk of SSIs and postoperative wound pain but a significantly better cosmetic effect was achieved. The use of staplers in suturing the skin shortens the duration of the surgical procedure; although it is associated with an increased risk of wound dehiscence in obese women. RR 5.2 (95\% CI 1.8-14.7) [30]. Similar results were obtained in a randomized cohort study involving 1147 women, in which sewing the skin with the staplers more than doubled the risk of postoperative wound dehiscence (RR 2.20; 95\% CI 1.6-3.1) and contributed to its infection (wound infection or cellulitis) (RR 2.46; 95\% CI 1.4-4.4) compared to the group of women who received intradermal sutures [28]. However, a 2018 study of women with grade III obesity did not confirm the above results [34].

\subsubsection{Subcutaneous Tissue Drainage}

The prophylactic drainage of subcutaneous tissue aims to reduce the risk of formation of fluid reservoirs within a wound, which could disrupt its continuity or lead to its infection. The studies conducted by Al-Inany et al. [22] and Ramsey et al. [23] did not confirm the effectiveness of the prophylactic drainage of subcutaneous tissue. Alanis, in a retrospective cohort study, concluded that prophylactic drainage of subcutaneous tissue should be abandoned in women with massive obesity [24]. An American randomized cohort study showed that the drainage procedure significantly increases the risk of wound complications (OR 2.86; 95\% CI 1.02-7.98), contributing to both its dehiscence and infection [27]. A study by Bindal and Munda based on obese women from India demonstrated that patients after a caesarean section with a drain had reduced rates of wound seroma, postoperative pain, and shorter hospital stays [35]. However, the authors did not observe any significant benefits of the drainage with regard to postoperative fever, superficial SSI, as well as wound 
breakdown [35]. A retrospective cohort study by Dias and colleagues [46] performed on severely obese women with a BMI of $>40 \mathrm{~kg} / \mathrm{m}^{2}$ showed no correlation between the use of a drain and SSI. A Japanese study analyzing two groups of women with a mean BMI of approx. $33 \mathrm{~kg} / \mathrm{m}^{2}$, the first of which had staples and the second had subcuticular sutures and drains [25] found that the frequency of wound complications was significantly lower in women with a drain as compared to those with staples. The authors used fourchannel Blake drains, which are made from soft fluted silicone with a wide surface area for drainage. This type of drain was demonstrated to be less painful in comparison to non-Blake drains [60]. On the other hand, Aziz Khalifa and colleagues reported a significant difference between obese women with a seroma drain after a caesarean section and women with no seroma drain $(9.6 \%$ vs. $26.7 \%$, respectively) and postoperative pain requiring analgesics [32]. Most of the available data regarding the usage of drains in post-caesarean women were obtained from studies performed on a low number of patients, which may have influenced the results. Thus, performing a meta-analysis may overcome the effect of a small analyzed population. To the best of our knowledge, no meta-analysis on the correlation between drainage use and wound complications in obese women after caesarean sections have been performed. The meta-analyses of Gates published in 2005 and 2013 [61] included studies analyzing women with both a normal BMI and overweight or obese women.

In the present study, we performed a meta-analysis concerning the comparisons of surgical complications after caesarean sections in obese women in terms of wound complications (including data on wound separation), infections (including data on SSI), as well as fevers.

In general, nine studies with a total number of 674 obese post caesarean women with a drain and 1718 obese women with no drain were included in the meta-analysis [21-25,27,32,35,46]. The impact of drainage on the risk of wound complications in obese women was conducted based on eight studies with a total number of 658 women with a drain and 1,283 obese women with no drain. Studies were highly heterogeneous ( $\left.\mathrm{I}^{2} 84 \%\right)$. The percentage of wound complications was slightly higher in obese women with a drain $(21 \%)$ compared to obese women without a drain (19\%). Since significant heterogeneity between studies was calculated, the REM method was used to calculate the pooled OR. The difference was not significant (OR 1.32; 95\% CI 0.64-2.70, $p=0.45$ ), which indicates that the use of drains does not increase the risk of wound complications in obese women after a caesarean section (Figure 3).

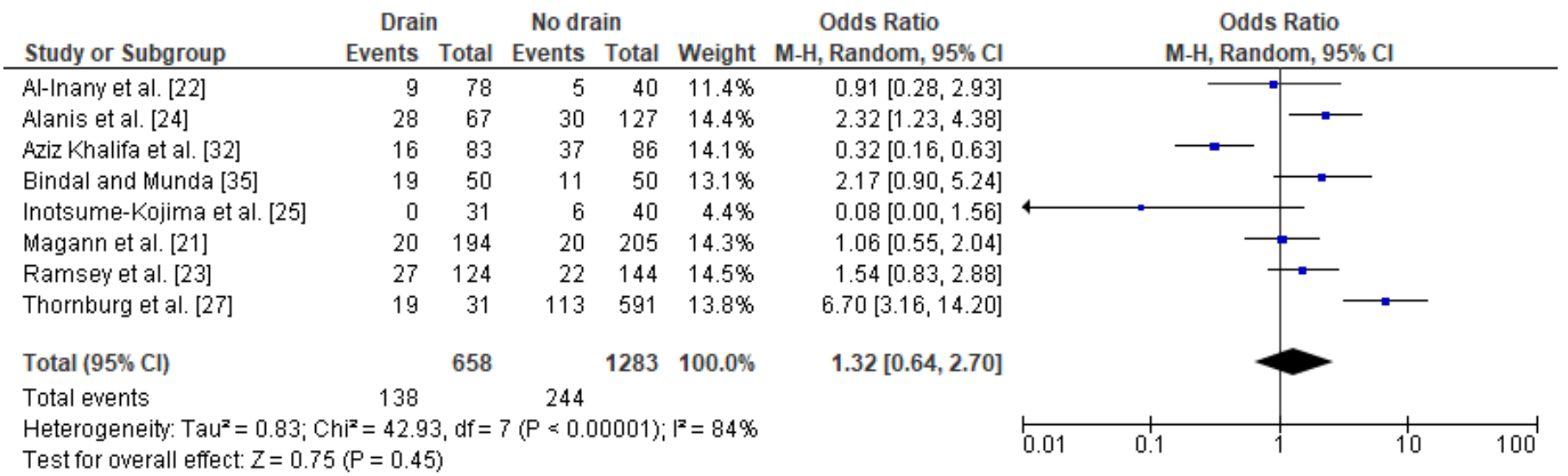

Figure 3. Forest plot for wound complications in the group of post-caesarean obese women with a drain and in the group of obese women without a drain. M.-H.—Mantel-Haenszel; CI—confidence interval; $\mathrm{I}^{2}$ — heterogeneity; df-degrees of freedom. 
The results were stable after subsequent omitting each of the studies that was included. No publication bias was found for this analysis [Egger's test $(p=0.577)$; Begg's test $(p=0.458)]$.

The relation between drainage and infections (including SSI) after a caesarean section in obese women was based on five studies with a total number of 258 women with a drain and 1202 obese women with no drain. The percentage of wound complications was slightly lower in obese women with a drain $(8 \%)$ as compared to obese women without a drain $(13 \%)$. The heterogeneity between studies were at low level $\left(\mathrm{I}^{2} 27 \%\right)$ with no significance, therefore FEM method was used to calculate the pooled OR. The difference was not significant (OR $0.93 ; 95 \%$ CI $0.53-1.63, p=0.80$ ), which indicates no impact of drain usage on infections after a caesarean section in obese women (Figure 4). The results were stable during the sensitivity analysis; thus, the analysis is reliable. Again, no publication bias was observed [Egger's test $(p=0.056)$; Begg's test $(p=0.142)]$.

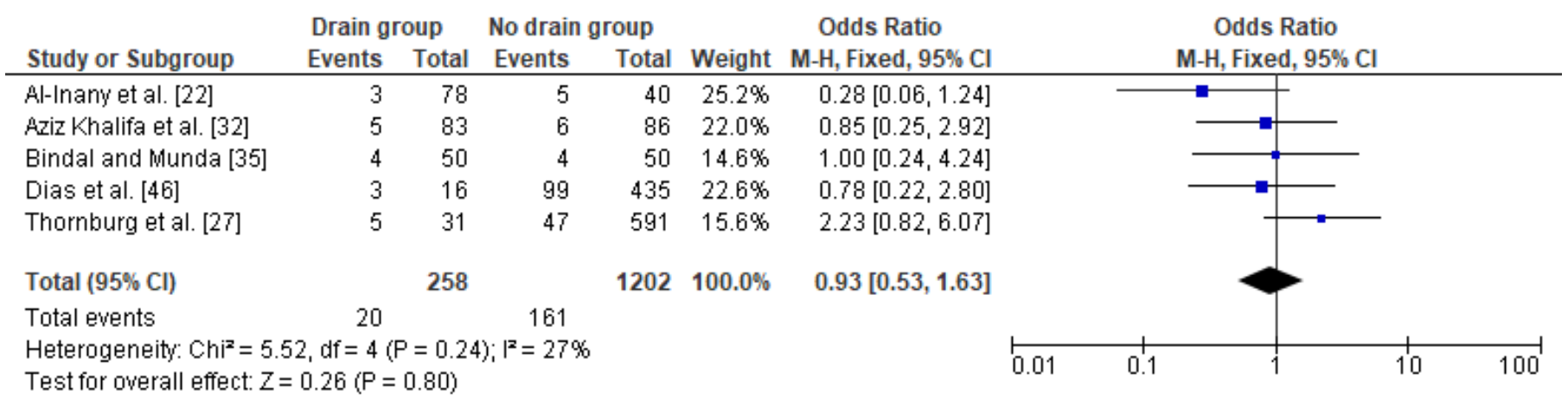

Figure 4. Forest plot for infections in the group of post-caesarean obese women with a drain and in the group of obese women without a drain. M.-H.-Mantel-Haenszel; CI—confidence interval; $\mathrm{I}^{2}$ —-heterogeneity; df-degrees of freedom.

In the case of the analysis of the impact of drainage on a fever after a caesarean section in obese women, three studies were included with a total number of 211 women with a drain and 176 obese women with no drain. The proportion of fevers was slightly lower in obese women with a drain (16\%) as compared to obese women without a drain (20\%). In this analysis, no heterogeneity between the studies was demonstrated $\left(\mathrm{I}^{2} 0 \%\right)$ and the FEM method was once again used to calculate the pooled OR. The difference was close to the border of significance (OR $0.62 ; 95 \%$ CI $0.36-1.07, p=0.09$ ) indicating that drain usage after a caesarean section may have some beneficial effect in the presence of a fever in obese women (Figure 5). The sensitivity analysis revealed that the results were stable and reliable and no publication bias was revealed [Egger's test $(p=0.342)$; Begg's test $(p=0.601)$ ].

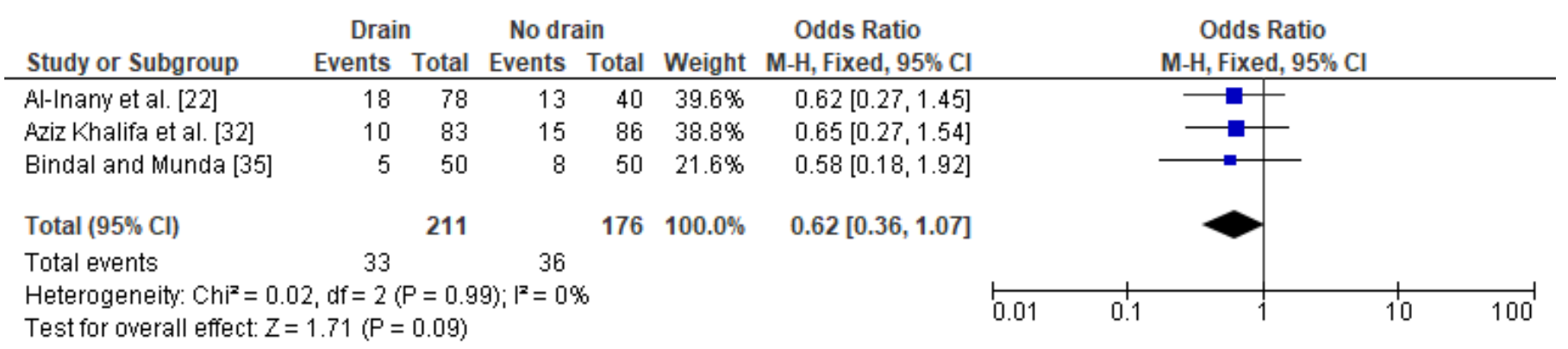

Figure 5. Forest plot for fever in the group of post-caesarean obese women with a drain and in the group of obese women without a drain. M.-H.-Mantel-Haenszel; CI—confidence interval; $\mathrm{I}^{2}$ —heterogeneity; df—degrees of freedom. 


\subsubsection{Negative Pressure Wound Therapy Dressings (VAC Dressings)}

Seven randomized trials concerning the effectiveness of vacuum dressings in the prevention of SSIs were carried out. Most of the studies indicated the ineffectiveness of the prophylactic use of the above therapy. The study conducted in women with a BMI of $\geq 30 \mathrm{~kg} / \mathrm{m}^{2}$ did not show the negative pressure wound therapy (NPWT) dressing to significantly reduce the incidence of complications in the postoperative wound compared to standard wound care ( 4.9 vs. $6.9 \% ; p=0.71)$ [37]. Similar results were provided by the Hussama study that included 441 women with morbid obesity (BMI $>40 \mathrm{~kg} / \mathrm{m}^{2}$ ), where a closed vacuum dressing was used as compared to standard wound care, and also did not significantly reduce the incidence of complications in the skin (RR 0.9 [95\% CI 0.5-1.4]) [43]. However, a Danish study from 2019 that encompassed 876 women with class I obesity or greater showed that SSIs were found in $4.6 \%$ in the NPWT dressing group, while in the control group there were $9.2 \%$ SSIs (RR $0.50 ; 95 \%$ CI $0.30-0.84$ ); the number needed to treat 22; $p=0.007$ ). The authors found that vacuum dressings used prophylactically in obese women significantly reduced the incidence of SSIs [43]. The results of the analysis are presented in Table 5.

Table 5. Results of studies included in the review and meta-analysis describing the role of prophylactic negative pressure therapy in surgical site infections.

\begin{tabular}{|c|c|c|c|c|c|}
\hline \multirow{2}{*}{ Study } & \multirow{2}{*}{$N$} & \multirow{2}{*}{ BMI $\left(\mathrm{kg} / \mathrm{m}^{2}\right)$} & \multicolumn{2}{|c|}{ Surgical Site Infection (SSI) } & \multirow{2}{*}{ Results } \\
\hline & & & $N$ & OR & \\
\hline Looby et al. [36] & 467 & $\geq 40$ & $\begin{array}{c}\text { NPWT 13/234 } \\
\text { SD 23/233 }\end{array}$ & $0.45(0.22-0.95)$ & $\begin{array}{c}\text { NPT after caesarean delivery in } \\
\text { women with a BMI of } \geq 40 \mathrm{~kg} / \mathrm{m}^{2} \\
\text { is an efficacious method to reduce } \\
\text { the incidence of postoperative } \\
\text { wound infections }\end{array}$ \\
\hline Ruhstaller et al. [37] & 119 & $\geq 30$ & $\begin{array}{l}\text { NPWT 3/61 } \\
\text { SD 4/58 }\end{array}$ & $0.70(0.15-3.26)$ & $\begin{array}{l}\text { Routine clinical use of a NPWT } \\
\text { system after a caesarean delivery } \\
\text { did not result in a significant } \\
\text { reduction in wound morbidity } \\
\text { over standard } \\
\text { wound complications }\end{array}$ \\
\hline Searle et al. [38] & 399 & $\geq 35$ & NPWT 36/399 & - & $\begin{array}{c}\text { Use of NPWT on closed surgical } \\
\text { incisions may be associated with } \\
\text { low incidence of SSI }\end{array}$ \\
\hline Wihbey et al. [39] & 166 & $\geq 35$ & $\begin{array}{c}\text { NPWT 25/80 } \\
\text { SD 29/86 }\end{array}$ & $0.89(0.47-1.71)$ & $\begin{array}{l}\text { There were no differences in the } \\
\text { occurrence of composite wound } \\
\text { complications between women } \\
\text { using prophylactic NPWT }\end{array}$ \\
\hline Hussamy et al. [43] & 441 & $\geq 40$ & $\begin{array}{l}\text { NPWT } 37 / 222 \\
\text { SD } 42 / 219\end{array}$ & $0.84(0.52-1.37)$ & $\begin{array}{l}\text { A NPWT device compared with a } \\
\text { standard dressing did not } \\
\text { significantly lower the wound } \\
\text { complication rate in morbidly } \\
\text { obese women undergoing } \\
\text { caesarean delivery }\end{array}$ \\
\hline Hylding et al. [44] & 876 & $\geq 30$ & $\begin{array}{c}\text { NPWT 20/432 } \\
\text { SD } 41 / 444\end{array}$ & $0.5(0.30-0.84)$ & $\begin{array}{l}\text { Prophylactic use of incisional } \\
\text { NPWT reduced the risk of } \\
\text { surgical site infections }\end{array}$ \\
\hline Tuuli et al. [47] & 1624 & $\geq 30$ & $\begin{array}{l}\text { NPWT 52/806 } \\
\text { SD 54/802 }\end{array}$ & $0.95(0.66-1.37)$ & $\begin{array}{l}\text { Prophylactic NPWT compared } \\
\text { with a standard wound dressing } \\
\text { did not significantly reduce the } \\
\text { risk of surgical-site infection }\end{array}$ \\
\hline
\end{tabular}

NPWT—negative pressure wound therapy, SD—standard therapy, $\mathrm{N} —$ number of cases, OR—odds ratio, BMI—body mass index.

Meta-analysis of the data regarding the frequency of SSI in obese women with NPWT in comparison to women with standard therapy was based on six studies with 1835 cases with NPWT therapy and 1842 patients with standard therapy. One of the studies was excluded because of incomplete data (absence of patient with standard therapy) [38]. This 
revealed that the prevalence of SSI was lower among obese women with NPWT than in women receiving standard wound therapy ( $8 \%$ vs. $10.5 \%$, respectively). A significant reduction in the SSI odds ratio was observed in obese women with negative pressure wound therapy compared to obese women with standard therapy (OR $=0.7695 \% \mathrm{CI}$ $0.60-0.95, p=0.02$ ) (Figure 6). Very low level of heterogeneity between the studies was observed $\left(\mathrm{I}^{2}=7 \%\right)$.

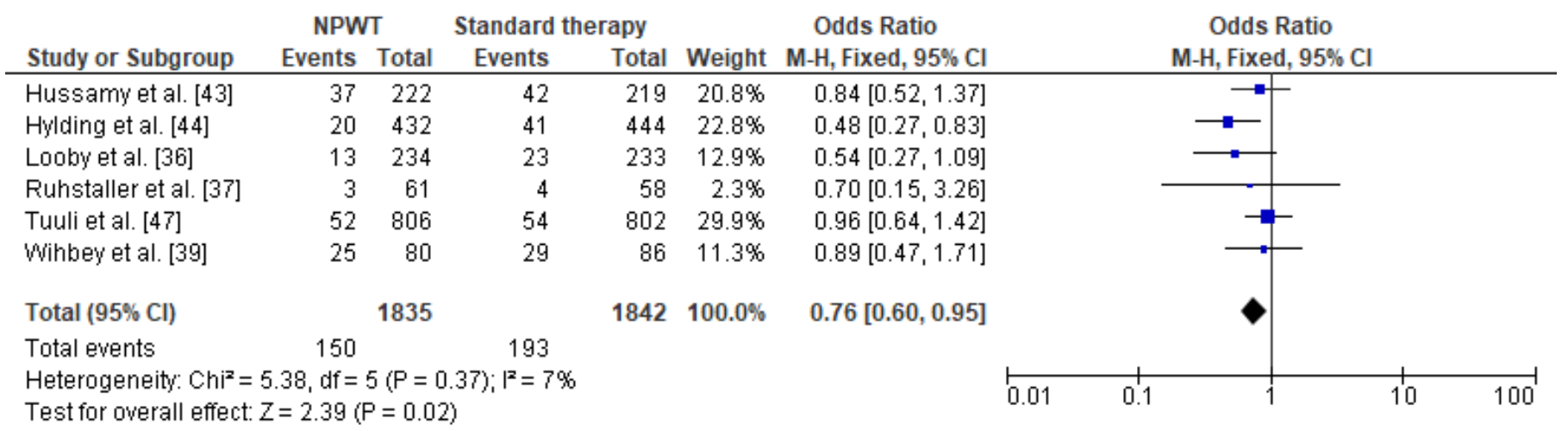

Figure 6. Forest plot for surgical site infection in the group of post-caesarean obese women with NPWT and in obese women with standard therapy. NPWT—negative pressure wound therapy; M.-H.-Mantel-Haenszel; CI-confidence interval; $\mathrm{I}^{2}$ - heterogeneity; df-degrees of freedom.

The results were stable after omitting one study at a time, i.e., the study by Hussamy et al. [43] first, then a study by Ruhstaller et al. [37], then a study by Wihbey et al. [39], and then a study by Tuuli et al. [47]. When the study by Hylding et al. [44] was omitted, the results changed and were not significant $(\mathrm{OR}=0.8495 \%$ CI $0.65-1.08, p=0.17)$. Similarly, omitting the data by Looby et al. [36] gave insignificant results ( $\mathrm{OR}=0.7995 \% \mathrm{CI} 0.62-1.01$, $p=0.06)$. Therefore the result of the meta-analysis should be treated with caution. The results of Egger's and Begg's tests showed no publication bias [Egger's test $(p=0.456)$; Begg's test $(p=0.348)]$.

\section{Discussion}

The use of a prophylactic dose of antibiotic in women undergoing a caesarean section reduces the risk of postoperative wound infections (RR 0.40; 95\% CI 0.35-0.46, 82) and inflammation of the endometrial mucosa (endometritis) (RR 0.38; 95\% CI 0.34-0.42, 83), as well as other complications resulting from infections (RR $0.31 ; 95 \%$ CI $0.20-0.49$ ) [62]. The time of administration of a prophylactic dose of antibiotics also affects the frequency of infection. The studies show that the administration of an antibiotic before a surgical procedure reduces the percentage of endometritis by $41 \%$ compared to the intraoperative administration of an antibiotic (RR 0.59; 95\% CI [95\% CI] 0.37-0.94; $\mathrm{I}^{2} 0 \%$ ), although such treatment does not reduce the incidence of postoperative wound infections (RR $0.71 ; 95 \%$ CI $0.44-1.14 ; \mathrm{I}^{2} 0 \%$ ) [63]. In clinical practice, the 1st-generation cephalosporin, Cefazolin, at a dose of $2 \mathrm{~g}$, which has a spectrum of activity including Gram-positive bacteria and Escherichia coli, is used in the prophylaxis of perioperative caesarean sections. As evidenced by studies conducted among obese women who were not pregnant, cefazolin shows different pharmacokinetics in obese people, which means that its concentration is lower in adipose tissue. This phenomenon suggests that the prophylactic dose needs to be increased in such a group of obese patients [64]. The studies that we analyzed did not confirm the effectiveness of a higher dose of antibiotic in perioperative prophylaxis.

The suturing of subcutaneous tissue aims to reduce the risk of formation of fluid reservoirs. A meta-analysis of six studies showed that suturing reduces the risk of wound complications by $34 \%$ [65] in subcutaneous tissue that is thicker than $2 \mathrm{~cm}$. A randomized study of 1082 women undergoing a caesarean section treated with antibiotic prophylaxis for more than $60 \mathrm{~min}$ from the skin incision, with the skin washed with an alcoholic 
chlorhexidine solution, and sutured subcutaneous tissue if its thickness was greater than 2 $\mathrm{cm}$, as well as skin closed with sutures instead of staplers, showed a significant reduction in the risk of complications in the postoperative wound, regardless of the method of incision of the skin, the coexistence of obesity and diabetes, chorioamnionitis or the operator's experience [11]. However, the studies analyzed by us and the meta-analysis on the method of incision of the skin demonstrated that a Pfannenstiel caesarean section reduces the risk of postoperative wound infections [66].

The prophylactic use of vacuum dressings in obese women is aimed at reducing the incidence of SSIs. As shown by a meta-analysis of seven studies, the prophylactic use of NPWT reduces the risk of SSIs (pooled RR 0.45; 95\% CI 0.31, 0.66). Complex wound complications were significantly reduced in patients receiving prophylactic negative pressure wound therapy compared to standard dressings (9 studies: pooled RR 0.68; 95\% CI $0.49,0.94$ ) [67]. Another meta-analysis conducted on 10 studies from 1966 to 2017 did not confirm that NPWT decreased wound complication (RR 0.97, 95 \% CI 0.63-1.49) [68]. Moreover the most recent American study based on 1624 people also did not significantly confirm NPWT in reducing SSIs. [47] The analysis carried out by us, supplemented with the current 6 researches, showed, that NPWT reduces the risk of SSIs, nevertheless, the results should be treated with caution because the sensitivity analysis was not stable and no significance was observed after omitting the studies by Hylding et al. [44] and Looby et al. [36] no significance was observed.

The prophylactic use of subcutaneous drains to reduce SSIs remains controversial. The meta-analysis concerning the use of subcutaneous tissue drainage after surgical procedures did not show a reduction in incidence of complications in the postoperative wounds; hence, it is unjustified in everyday surgical practice [69], as well as in caesarean sections and other surgical procedures performed in obese patients [70], which was confirmed in our meta-analysis.

The strength of our study is the meta-analysis of the research on the prophylactic use of a subcutaneous drain during a caesarean section procedure in obese women. It should be noted that no such analysis has been carried out to date. In addition, current research on the NPWT confirms that SSIs are significantly infrequent in obese women with NPWT, however, further analysis should provide more studies to confirm stable and reliable results.

The relatively small number of studies and significant variability in outcome reporting are important limitations of our study. All analyzed studies by us were in English language, which also might be a limitation of the study. Moreover, a considerable amount of research concerning the issue of caesarean sections includes retrospective studies that do not comprise clinical indications for a surgical procedure and do not refer to data regarding the duration of amniotic fluid leakage, the presence of uterine contractions, and a history of diabetes. Furthermore, the authors of some studies did not differentiate between caesarean sections in schedule and emergency ones $[10,26,34,41]$. The heterogeneity of the studies included in the review also constitutes a limitation of the study.

\section{Conclusions}

This paper studies the optimal management of a caesarean section wound including a transverse incision of the skin. NPWT reduces risk of surgical site infection. On the other hand subcutaneous drain does not reduce the risk of wound complications, wound infections, and fever in obese women after a caesarean section. The optimal dose of antibiotics in perioperative prophylaxis is still under investigation; therefore, it is advisable to conduct further multicenter studies on the caesarean section procedure in obese women.

Supplementary Materials: The following are available online at https:/ / www.mdpi.com/2077-038 3/10/4/675/s1. Table S1: Jadad's scale.

Author Contributions: All the authors qualified for authorship by having contributed substantially to the present study. A.S.-J. contributed to the study conceptualization, data collection and analysis, 
and manuscript writing. J.K.S., Ł.J. contributed to the data collection. B.S.-H. contributed to the data collection and statistical analyses. All the authors have approved the submitted version and have agreed to be accountable for all its contents. All authors have read and agreed to the published version of the manuscript.

Funding: This research received no external funding.

Conflicts of Interest: The authors declare that they have no conflict of interest.

\section{References}

1. Sepúlveda, J.; Murray, C. The State of Global Health in 2014. Science 2014, 345, 1275-1278. [CrossRef]

2. World Health Organization. Obesity and Overweight. Available online: https://www.who.int/news-room/fact-sheets/detail/ obesity-and-overweight (accessed on 7 June 2019).

3. Ogden, C.L.; Carroll, M.D.; Curtin, L.R.; McDowell, M.A.; Tabak, C.J.; Flegal, K.M. Prevalence of Overweight and Obesity in the United States, 1999-2004. JAMA 2006, 295, 1549-1555. [CrossRef]

4. Shaikh, H.; Robinson, S.; Teoh, T.G. Management of Maternal Obesity Prior to and During Pregnancy. Semin. Fetal. Neonatal. Med. 2010, 15, 77-82. [CrossRef]

5. $\quad$ Feig, D.S.; Hwee, J.; Shah, B.R.; Booth, G.L.; Bierman, A.S.; Lipscombe, L.L. Trends in Incidence of Diabetes in Pregnancy and Serious Perinatal Outcomes: A Large, Population-Based Study in Ontario, Canada, 1996-2010. Diabetes Care. 2014, 37, 1590-1596. [CrossRef]

6. Crane, J.M.G.; White, J.; Murphy, P.; Burrage, L.; Hutchens, D. The Effect of Gestational Weight Gain by Body Mass Index on Maternal and Neonatal Outcomes. J. Obstet. Gynaecol. Can. 2009, 31, 28-35. [CrossRef]

7. Quiner, T.; Perlow, J.H. The Global Burden of Obesity on Pregnancy Outcomes in the Developed World [17Q]. Obstet. Gynecol. 2017, 1, 177S. [CrossRef]

8. Vaswani, P.R.; Balachandran, L. Pregnancy Outcomes in a Population with High Prevalence of Obesity: How bad is it? Clin. Epidemiol. Glob. Health 2013, 1, 5-11. [CrossRef]

9. Marchi, J.; Berg, M.; Dencker, A.; Olander, E.K.; Begley, C. Risks Associated with Obesity in Pregnancy, for the Mother and Baby: A Systematic Review of Reviews. Obes. Rev. 2015, 16, 621-638. [CrossRef] [PubMed]

10. Conner, S.N.; Verticchio, J.C.; Tuuli, M.G.; Odibo, A.O.; Macones, G.A.; Cahill, A.G. Maternal Obesity and Risk of Postcesarean Wound Complications. Am. J. Perinatol. 2014, 31, 299-304.

11. Temming, L.A.; Raghuraman, N.; Carter, E.B.; Stout, M.J.; Rampersad, R.M.; Macones, G.A.; Cahill, A.G.; Tuuli, M.G. Impact of Evidence-Based Interventions on Wound Complications after Cesarean Delivery. Am. J. Obstet. Gynecol. 2017, 217, 449.e1-449.e9. [CrossRef]

12. Salim, R.; Braverman, M.; Teitler, N.; Berkovic, I.; Suliman, A.; Shalev, E. Risk Factors for Infection Following Cesarean Delivery: An Interventional Study. J. Matern. Fetal Neonatal. Med. 2012, 25, 2708-2712. [CrossRef] [PubMed]

13. Conroy, K.; Koenig, A.F.; Yu, Y.H.; Courtney, A.; Lee, H.J.; Norwitz, E.R. Infectious Morbidity after Cesarean Delivery: 10 Strategies to Reduce Risk. Rev. Obstet. Gynecol. 2012, 5, 69-77. [PubMed]

14. Mackeen, A.D.; Schuster, M.; Berghella, V. Suture versus Staples for Skin Closure after Cesarean: A Metaanalysis. Am. J. Obstet. Gynecol. 2015, 212, 621.e1-621.e10. [CrossRef]

15. Kawakita, T.; Landy, H.J. Surgical Site Infections after Cesarean Delivery: Epidemiology, Prevention and Treatment. Matern. Health Neonatol. Perinatol. 2017, 3, 12. [CrossRef]

16. Moher, D.; Shamseer, L.; Clarke, M.; Ghersi, D.; Liberati, A.; Petticrew, M.; Shekelle, P.; Stewart, L. Preferred Reporting Items for Systematic Review and Meta-analysis Protocols (PRISMA-P). Syst. Rev. 2015, 4, 1. [CrossRef] [PubMed]

17. Sanderson, S.; Tatt, I.D.; Higgins, J.P.T. Tools for Assessing Quality and Susceptibility to Bias in Observational Studies in Epidemiology: A Systematic Review and Annotated Bibliography. Int. J. Epidemiol. 2007, 36, 666-676. [CrossRef]

18. Jadad, A.R.; Moore, R.A.; Carroll, D.; Jenkinson, C.; Reynolds, D.J.M.; Gavaghan, D.J.; McQuay, H.J. Assessing the Quality of Reports of Randomized Clinical Trials: Is Blinding Necessary? Control. Clin. Trials 1996, 17, 1-12. [CrossRef]

19. Egger, M.; Davey Smith, G.; Schneider, M.; Minder, C. Bias in Meta-Analysis Detected by a Simple, Graphical Test. BMJ. 1997, 315, 629-634. [CrossRef]

20. Sterne, J.A.; Sutton, A.J.; Ioannidis, J.P.; Terrin, N.; Jones, D.R.; Lau, J.; Carpenter, J.; Rücker, G.; Harbord, R.M.; Schmid, C.H.; et al. Recommendations for Examining and Interpreting Funnel Plot Asymmetry in Meta-analyses of Randomised Controlled Trials. BMJ 2011, 343, d4002. [CrossRef]

21. Magann, E.F.; Chauhan, S.P.; Rodts-Palenik, S.; Bufkin, L.; Martin, J.N., Jr.; Morrison, J.C. Subcutaneous Stitch Closure versus Subcutaneous Drain to Prevent Wound Disruption after Cesarean Delivery: A Randomized Clinical Trial. Am. J. Obstet. Gynecol. 2002, 186, 1119-1123. [CrossRef] [PubMed]

22. Al-Inany, H.; Youssef, G.; Abd ElMaguid, A.; Hamid, M.A.; Naguib, A. Value of Subcutaneous Drainage System in Obese Females Undergoing Cesarean Section Using Pfannenstiel Incision. Gynecol. Obstet. Investig. 2002, 53, 75-78. [CrossRef]

23. Ramsey, P.S.; White, A.M.; Guinn, D.A.; Lu, G.C.; Ramin, S.M.; Davies, J.K.; Neely, C.L.; Newby, C.; Fonseca, L.; Case, A.S.; et al. Subcutaneous Tissue Reapproximation, Alone or in Combination with Drain, in Obese Women Undergoing Cesarean Delivery. Obstet. Gynecol. 2005, 105, 967-973. [CrossRef] 
24. Alanis, M.C.; Villers, M.S.; Law, T.L.; Steadman, E.M.; Robinson, C.J. Complications of Cesarean Delivery in the Massively Obese Parturient. Am. J. Obstet. Gynecol. 2010, 203, 271.e1-271.e7. [CrossRef]

25. Inotsume-Kojima, Y.; Uchida, T.; Abe, M.; Doi, T.; Kanayama, N. A Combination of Subcuticular Sutures and a Drain for Skin Closure Reduces Wound Complications in Obese Women Undergoing Surgery Using Vertical Incisions. J. Hosp. Infect. 2011, 77, 162-165. [CrossRef] [PubMed]

26. McLean, M.; Hines, R.; Polinkovsky, M.; Stuebe, A.; Thorp, J.; Strauss, R. Type of Skin Incision and Wound Complications in the Obese Parturient. Am. J. Perinatol. 2012, 29, 301-306. [CrossRef]

27. Thornburg, L.L.; Linder, M.A.; Durie, D.E.; Walker, B.; Pressman, E.; Glantz, J.C. Risk Factors for Wound Complications in Morbidly Obese Women Undergoing Primary Cesarean Delivery. J. Matern. Fetal. Neonatal. Med. 2012, 25, 1544-1548. [CrossRef] [PubMed]

28. Subramaniam, A.; Jauk, V.C.; Figueroa, D.; Biggio, J.R.; Owen, J.; Tita, A.T. Risk Factors for Wound Disruption Following Cesarean Delivery. J. Matern. Fetal. Neonatal Med. 2014, 27, 1237-1240. [CrossRef]

29. Stamilio, D.M.; Scifres, C.M. Extreme Obesity and Postcesarean Maternal Complications. Obstet. Gynecol. 2014, 124, $227-232$. [CrossRef]

30. Ibrahim, M.I.; Moustafa, G.F.; Al-Hamid, A.S.; Hussein, M.R. Superficial Incisional Surgical Site Infection Rate after Cesarean Section in Obese Women: A Randomized Controlled Trial of Subcuticular Versus Interrupted Skin Suturing. Arch. Gynecol. Obstet. 2014, 289, 981-986. [CrossRef] [PubMed]

31. Smid, M.C.; Kearney, M.S.; Stamilio, D.M. Extreme Obesity and Postcesarean Wound Complications in the Maternal-Fetal Medicine Unit Cesarean Registry. Am. J. Perinatol. 2015, 32, 1336-1341. [CrossRef]

32. Khalifa, A.A.A.; Abdelrazak, K.M.; Abdelazim, I.A. Routine Subcutaneous Drain versus No Drain in Cesarean Section for Diabetic Obese Women: A Randomized Controlled Trial. Int. J. Curr. Microbiol. App. Sci. 2015, 4, 479-485.

33. Sutton, A.L.; Sanders, L.B.; Subramaniam, A.; Jauk, V.C.; Edwards, R.K. Abdominal Incision Selection for Cesarean Delivery of Women with Class III Obesity. Am J Perinatol. 2016, 33, 547-551. [CrossRef]

34. Zaki, M.N.; Truong, M.; Pyra, M.; Kominiarek, M.A.; Irwin, T. Wound Complications in Obese Women after Cesarean: A Comparison of Staples versus Subcuticular Suture. J. Perinatol. 2016, 36, 819-822. [CrossRef] [PubMed]

35. Bindal, J.A.; Munda, G. Clinical Study to Compare Drain versus No Drain in Post Cesarean Section. Int. J. Reprod. Contracept. Obstet. Gynecol. 2017, 6, 3903-3906. [CrossRef]

36. Looby, M.A.; Vogel, R.I.; Bangdiwala, A.; Hyer, B.; Das, K. Prophylactic Negative Pressure Wound Therapy in Obese Patients Following Cesarean Delivery. Surg. Innov. 2018, 25, 43-49. [CrossRef] [PubMed]

37. Ruhstaller, K.; Downes, K.L.; Chandrasekaran, S.; Srinivas, S.; Durnwald, C. Prophylactic Wound Vacuum Therapy after Cesarean Section to Prevent Wound Complications in the Obese Population: A Randomized Controlled Trial (the ProVac Study). Am. J. Perinat. 2017, 34, 1125-1130. [CrossRef] [PubMed]

38. Searle, R.J.; Myers, D. A Survey of Caesarean Section Surgical Site Infections with PICO ${ }^{\text {TM }}$ Single Use Negative Pressure Wound Therapy System in High-Risk Patients in England and Ireland. J. Hosp. Infect. 2017, 97, 122-124. [CrossRef]

39. Wihbey, K.A.; Joyce, E.M.; Spalding, Z.T.; Jones, H.J.; MacKenzie, T.A.; Evans, R.H.; Fung, J.L.; Goldman, M.B.; Erekson, E. Prophylactic Negative Pressure Wound Therapy and Wound Complication After Cesarean Delivery in Women With Class II or III Obesity: A Randomized Controlled Trial. Obstet. Gynecol. 2018, 132, 377-384. [CrossRef] [PubMed]

40. Zaki, M.N.; Wing, D.A.; McNulty, J.A. Comparison of Staples vs Subcuticular Suture in Class III Obese Women Undergoing Cesarean: A Randomized Controlled Trial. Am. J. Obstet. Gynecol. 2018, 218, 451.e1-451.e8. [CrossRef]

41. Alalfy, M.; Elgazzar, A.; Fares, T.; Nagy, O.; Ellithy, A.; Lasheen, Y.; Kamel, A.; Soliman, M.; Hassan, A.; Samy, A.; et al. Effect of Subcutaneous Tissue Closure Technique in Cesarean Section on Postoperative Wound Complications in Obese Egyptian Women. J. Matern. Fetal. Neonatal. Med. 2019, 32, 2452-2459. [CrossRef]

42. Marrs, C.; Blackwell, S.; Hester, A.; Olson, G.; Saade, G.R.; Faro, J.; Pedroza, C.; Sibai, B. Pfannenstiel versus Vertical Skin Incision for Cesarean Delivery in Women with Class III Obesity: A Randomized Trial. Am. J. Perinatol. 2019, 36, 97-104.

43. Hussamy, D.J.; Wortman, A.C.; McIntire, D.D.; Leveno, K.J.; Casey, B.M.; Roberts, S.W. Closed Incision Negative Pressure Therapy in Morbidly Obese Women Undergoing Cesarean Delivery: A Randomized Controlled Trial. Obstet. Gynecol. 2019, 134, 781-789. [CrossRef]

44. Hyldig, N.; Vinter, C.A.; Kruse, M.; Mogensen, O.; Bille, C.; Sorensen, J.A.; Lamont, R.F.; Wu, C.; Heidemann, L.N.; Ibsen, M.H.; et al. Prophylactic Incisional Negative Pressure Wound Therapy Reduces the Risk of Surgical Site Infection after Caesarean Section in Obese Women: A Pragmatic Randomised Clinical Trial. Int. J. Obstet. Gynaecol. 2019, 126, 628-635. [CrossRef]

45. Connery, S.A.; Yankowitz, J.; Odibo, L.; Raitano, O.; Nikolic-Dorschel, D.; Louis, J.M. Effect of Using Silver Nylon Dressings to Prevent Superficial Surgical Site Infection after Cesarean Delivery: A Randomized Clinical Trial. Am. J. Obstet. Gynecol. 2019, 221, 57.e1-57.e7. [CrossRef] [PubMed]

46. Dias, M.; Dick, A.; Reynolds, R.M.; Lahti-Pulkkinen, M.; Denison, F.C. Predictors of Surgical Site Skin Infection and Clinical Outcome at Caesarean Section in the Very Severely Obese: A Retrospective Cohort Study. PLoS ONE 2019, 14, e0216157. [CrossRef]

47. Tuuli, M.G.; Liu, J.; Tita, A.T.N.; Longo, S.; Trudell, A.; Carter, E.B.; Shanks, A.; Woolfolk, C.; Caughey, A.B.; Warren, D.K.; et al. Effect of Prophylactic Negative Pressure Wound Therapy vs Standard Wound Dressing on Surgical-Site Infection in Obese Women After Cesarean Delivery: A Randomized Clinical Trial. JAMA 2020. [CrossRef] 
48. Pevzner, L.; Swank, M.; Krepel, C.; Wing, D.A.; Chan, K.; Edmiston, C.E., Jr. Effects of Maternal Obesity on Tissue Concentrations of Prophylactic Cefazolin During Cesarean Delivery. Obstet. Gynecol. 2011, 117, 877-882. [CrossRef] [PubMed]

49. Stitely, M.; Sweet, M.; Slain, D.; Alons, L.; Holls, W.; Hochberg, C.; Briggs, F. Plasma and Tissue Cefazolin Concentrations in Obese Patients Undergoing Cesarean Delivery and Receiving Differing Pre-operative Doses of Drug. Surg. Infect. 2013, 14, 455-459. [CrossRef] [PubMed]

50. Ahmadzia, H.K.; Patel, E.M.; Joshi, D.; Liao, C.; Witter, F.; Heine, R.P.; Coleman, J.S. Obstetric Surgical Site Infections: 2 grams Compared with 3 grams of Cefazolin in Morbidly Obese Women. Obstet. Gynecol. 2015, 126, 708-715. [CrossRef]

51. Young, O.M.; Shaik, I.H.; Twedt, R.; Binstock, A.; Althouse, A.D.; Venkataramanan, R.; Simhan, H.N.; Wiesenfeld, H.C.; Caritis, S.N. Pharmacokinetics of Cefazolin Prophylaxis in Obese Gravidae at Time of Cesarean Delivery. Am. J. Obstet. Gynecol. 2015, 213, 541.e1-541.e7. [CrossRef] [PubMed]

52. Swank, M.L.; Wing, D.A.; Nicolau, D.P.; McNulty, J.A. Increased 3-gram Cefazolin Dosing for Cesarean Delivery Prophylaxis in Obese Women. Am. J. Obstet. Gynecol. 2015, 213, 415.e1-415.e8. [CrossRef]

53. Maggio, L.; Nicolau, D.P.; DaCosta, M.; Rouse, D.J.; Hughes, B.L. Cefazolin Prophylaxis in Obese Women Undergoing Cesarean Delivery: A Randomized Controlled Trial. Obstet. Gynecol. 2015, 125, 1205-1210. [CrossRef]

54. Lillico, R.; Sayre, C.L.; Sitar, D.S.; Davies, N.M.; Baron, C.M.; Lakowski, T.M. Quantification of Cefazolin in Serum and Adipose Tissue by Ultra High Performance Liquid Chromatography-Tandem Mass Spectrometry (UHPLC-MS/MS): Application to a Pilot Study of Obese Women Undergoing Cesarean Delivery. J. Chromatogr. B. Analyt. Technol. Biomed. Life Sci. 2016, 1031, 94-98. [CrossRef]

55. Groff, S.M.; Fallatah, W.; Yang, S.; Murphy, J.; Crutchfield, C.; Marzinke, M.; Kurtzberg, J.; Lee, C.K.K.; Burd, I.; Farzin, A. Effect of Maternal Obesity on Maternal-Fetal Transfer of Preoperative Cefazolin at Cesarean Section. J. Pediatr. Pharmacol. Ther. 2017, 22, 227-232. [CrossRef]

56. Grupper, M.; Kuti, J.L.; Swank, M.L.; Maggio, L.; Hughes, B.L.; Nicolau, D.P. Population Pharmacokinetics of Cefazolin in Serum and Adipose Tissue from Overweight and Obese Women Undergoing Cesarean Delivery. J. Clin. Pharmacol. 2017, 7, 712-719. [CrossRef] [PubMed]

57. Kram, J.J.F.; Greer, D.M.; Cabrera, O.; Burlage, R.; Forgie, M.M.; Siddiqui, D.S. Does Current Cefazolin Dosing Achieve Adequate Tissue and Blood Concentrations in Obese Women Undergoing Cesarean Section? Eur. J. Obstet. Gynecol. Reprod. Biol. 2017, 210, 334-341. [CrossRef] [PubMed]

58. Eley, V.A.; Christensen, R.; Ryan, R.; Jackson, D.; Parker, S.L.; Smith, M.; van Zundert, A.A.; Wallis, S.C.; Lipman, J.; Roberts, J.A. Prophylactic Cefazolin Dosing in Women with Body Mass Index $>35 \mathrm{~kg} \cdot \mathrm{m}^{2}$ Undergoing Cesarean Delivery: A Pharmacokinetic Study of Plasma and Interstitial Fluid. Anesth. Analg. 2020, 131, 199-207. [CrossRef]

59. Scheck, S.M.; Blackmore, T.; Maharaj, D.; Langdana, F.; Elder, R.E. Caesarean Section Wound Infection Surveillance: Information for Action. Aust. N. Z. J. Obstet. Gynaecol. 2018, 58, 518-524. [CrossRef]

60. Rayatt, S.S.; Dancey, A.L.; Jaffe, W. Soft Fluted Silicone Drains: A Prospective, Randomized, Patient-Controlled Study. Plast. Reconstr. Surg. 2005, 115, 1605-1608. [CrossRef] [PubMed]

61. Hellums, E.K.; Lin, M.G.; Ramsey, P.S. Prophylactic Subcutaneous Drainage for Prevention of Wound Complications after Cesarean Delivery-A Metaanalysis. Am. J. Obstet. Gynecol. 2007, 197, 229-235. [CrossRef] [PubMed]

62. Smaill, F.M.; Grivell, R.M. Antibiotic Prophylaxis versus No Prophylaxis for Preventing Infection after Cesarean Section. Cochrane Database Syst. Rev. 2014, 28, CD007482. [CrossRef]

63. Baaqeel, H.; Baaqeel, R. Timing of Administration of Prophylactic Antibiotics for Caesarean Section: A Systematic Review and Meta-analysis. BJOG 2013, 120, 661-669. [CrossRef] [PubMed]

64. Toma, O.; Suntrup, P.; Stefanescu, A.; London, A.; Mutch, M.; Kharasch, E. Pharmacokinetics and Tissue Penetration of Cefoxitin in Obesity: Implications for Risk of Surgical Site Infection. Anesth. Analg. 2011, 113, 730e7. [CrossRef] [PubMed]

65. Chelmow, D.; Rodriguez, E.J.; Sabatini, M.M. Suture Closure of Subcutaneous Fat and Wound Disruption after Cesarean Delivery: A Meta-analysis. Obstet. Gynecol. 2004, 103, 974e80. [CrossRef]

66. Mccurdy, R.J.; Felder, L.A.; Saccone, G.; Edwards, R.K.; Thornburg, L.L.; Marrs, C.; Conner, S.N.; Strauss, R.; Berghella, V. The Association of Skin Incision Placement During Cesarean Delivery with Wound Complications in Obese Women: A Systematic Review and Meta-analysis. J. Matern. Fetal. Neonatal. Med. 2020, 7, 1-13. [CrossRef]

67. Yu, L.; Kronen, R.J.; Simon, L.E.; Stoll, C.R.T.; Colditz, G.A.; Tuuli, M.G. Prophylactic Negative-Pressure Wound Therapy after Cesarean is Associated with Reduced Risk of Surgical Site Infection: A Systematic Review and Meta-analysis. Am. J. Obstet. Gynecol. 2018, 218, 200-210. [CrossRef] [PubMed]

68. Smid, M.C.; Dotters-Katz, S.K.; Grace, M.; Wright, S.T.; Villers, M.S.; Hardy-Fairbanks, A.; Stamilio, D.M. Prophylactic Negative Pressure Wound Therapy for Obese Women After Cesarean Delivery: A Systematic Review and Meta-analysis. Obstet. Gynecol. 2017, 130, 969-978. [CrossRef] [PubMed]

69. Coletta, D.; Del Basso, C.; Giuliani, G.; Guerra, F. Subcutaneous Suction Drains Do Not Prevent Surgical Site Infections in Clean-Contaminated Abdominal Surgery-Results of a Systematic Review and Meta-analysis. Langenbecks Arch. Surg. 2019, 404, 663-668. [CrossRef]

70. Kosins, A.M.; Scholz, T.; Cetinkaya, M.; Evans, G.R. Evidence-Based Value of Subcutaneous Surgical Wound Drainage: The Largest Systematic Review and Meta-analysis. Plast. Reconstr. Surg. 2013, 132, 443-450. [CrossRef] 